\title{
Sui sistemi di integrali indipendenti di una equazione lineare ed omogenea a derivate parziali di $10^{\circ}$ ordine.
}

\author{
(Memoria del prof. G. Rrcer, a Padova.)
}

\section{INTRODUZIONE.}

11 problema dei sistemi ortogonali in una varietà ad $n$ dimensioni ̀̀ suscettibile di una generalizzazione, a cui, per quanto so, non è ancora stato posto mente e che forma l'oggetto delle ricerche qui svolte.

Si tratta di trovare le condizioni necessarie e sufficienti perchè una equazione a derivate parziali di $1 .^{\circ}$ ordine lineare ed omogenea con $n$ variabili indipendenti $x_{1}, x_{2}, \ldots x_{n}$ ammetta un sistema di $n-1$ integrali ortogonali fra di loro in una varietà ad $n$ dimensioni, di cui è dato l'elemento lineare, e i cui punti si considerano determinati mediante quelle variabili; e verificate quelle condizioni riconoscere se e quanto gli integrali stessi siano determinati.

Se con $Y_{r}$ si indica il coefficiente della derivata della funzione incognita rispetto ad $x_{r}$ nella equazione a derivate parziali, con $a_{r s}$ quello di $d x_{r} d x_{s}$ nel quadrato dell'elemento lineare e, posto $X_{r}=\Sigma_{s} a_{r s} Y_{s}$, si suppone che le $X_{r}$ siano proporzionali alle derivate di una stessa funzione rispetto alle $x_{r}$, il problema enunciato coincide con quello dei sistemi ortogonali nella varietà, di cui si tratta. Se si suppone di più che l'espressione del quadrato dell'elemento lineare di questa con un cambiamento di variabili possa mettersi sotto la forma $\sum_{1}^{n} d y_{r}^{2}$, ciò̀ che, secondo una classificazione da me proposta, quella espressione sia una forma quadratica differenziale di classe 0 , il problema stesso è quello studiato dal Darboux nella sua bella Memoria pubblicata nel Volume VII della Serie 2." delle Annales de l'École Normale Supérieure, colla differenza che le equazioni, a cui noi giungeremo per questo caso, si riferiscono ad un sistema qualunque di variabili e non soltanto a quello, che dà al quadrato dell'elemento lineare la forma sopra indicata. 
Anche prescindendo da queste speciali applicazioni, la cui importanza ̀̀ ben nota, parmi che il problema enunciato e i risultati ottenuti abbiano un interesse loro proprio come quelli, che mettono in evidenza un nuovo aspetto della teoria delle equazioni lineari ed omogenee a derivate parziali di $1 .^{\circ}$ ordine e costituiscono in casi molto generali una riduzione notevole del problema della loro integrazione.

Da un mio recente lavoro $\left(^{*}\right)$ appare già come l'unire la considerazione di una forma differenziale quadratica ad $n$ variabili allo studio delle funzioni di queste introduca nelle formule, che servono pel cambiamento di variabili nel calcolo differenziale, non soltanto una maggiore simmetria, ma ben'anche una maggiore generalità, di cui si può trarre partito per mettere le equazioni di dati problemi sotto la forma, che apparirà più opportuna per la loro soluzione. Ne avremo qui un esempio poichè vedremo che il problema della integrazione di una equazione lineare ed omogenea a derivate parziali di 1. ${ }^{\circ}$ ordine presenta maggiore o minore difficoltà secondo la natura della forma quadratica differenziale, che vi si connette, il che potrà convincere qualcuno dell' aiuto, che si può trarre in altri ed importanti campi di ricerca dallo studio di dette forme.

Quando si cerca di tradurre il problema proposto in linguaggio analitico, si incontra dapprima, come vedremo nel $\S 2$, una equazione algebrica di grado $n-1$, i cui coefficienti dipendono tanto dalle $Y_{r}$ quanto dalle $a_{r s}$ e dalle derivate prime delle une e delle altre. Essa ba forma analoga a quella, che dà nel nostro spazio le curvature principali di un sistema di superficie e ne è la generalizzazione. Mediante alcune formule dimostrate nel $\S 1$ si vede facilmente che le radici di detta equazione non dipendono affatto dalla scelta delle coordinate, ma soltanto dalla natura della varietà ad $n$ dimensioni e da quella della equazione a derivate parziali. Potremo quindi chiamarla equazione algebrica caratteristica di questa equazione in quella varietà.

Ne] $\S 3$ consideriamo il caso in cui le radici $\omega_{1}, \omega_{2}, \ldots \omega_{n-1}$ della equazione algebrica caratteristica, le quali sono sempre reali, siano anche tutte disuguali. A una qualunque di esse $\omega_{u}$ corrisponde allora un sistema di $n$ quantità $\mathrm{H}_{r u}(r=1,2, . ., n)$ e se poniamo $Y_{r}=\mathrm{H}_{r n}$ le linee di equazioni:

$$
\frac{d x_{1}}{\Pi_{1 u}}=\frac{d x_{2}}{\mathrm{H}_{2 u}}=\cdots=\frac{d x_{n}}{\mathrm{H}_{3 z}} \quad(u=1,2, \ldots n)
$$

(*) Sui parametri e gli invarianti delle forme quadratiche differenziali (Tomo XIV della Serie 2." di questi Annali). 
sono ortogonali fra di loro due a due. Le condizioni necessarie e sufficienti per l'esistenza degli $n-1$ integrali ortogonali cercati sono allora quelle necessarie e sufficienti perchè, posto $\Xi_{r u}=\Sigma_{s} a_{r s} \mathrm{H}_{s u}$, ciascuna delle $n-1$ equazioni differenziali totali $\sum_{r} \Xi_{r u} d x_{r}=0,(u=1,2, \ldots n-1)$ sia integrabile con una sola primitiva $\rho_{u}=$ costante e, quando così è, $\rho_{1}, \rho_{2}, \ldots \rho_{n-1}$ costituiscono appunto il sistema cercato di integrali della equazione proposta ortogonali fra di loro due a due nella varietà data; sistema, che è quindi in questo caso oompletamente determinato. Se assieme alla equazione proposta stessa consideriamo le equazioni pure lineari ed omogenee a derivate parziali di $10^{\circ}$ ordine di coefficienti $\mathrm{H}_{r u}$ le condizioni indicate equivalgono alle seguenti: $10^{\circ}$ che siano completi gli $n-1$ sistemi di equazioni, che si hanno combinando la proposta con una qualunque di quelle di coefficienti $\mathrm{H}_{r u} ; 2 .^{\circ}$ che siano completi gli $\frac{(n-1)(n-2)}{2}$ sistemi di equazioni, che risultano della proposta e di due di queste ultime equazioni. Abbiamo cosl due sistemi di equazioni di condizione affatto indipendenti l'uno dall'altro, e di cui l'uno $S_{1}$ ne comprende $\frac{(n-1)(n-2)}{2}$, l'altro $S_{z}$ ne comprende $\frac{(n-1)(n-2)(n-3)}{2}$. Queste ultime però si riducono' ad $\frac{(n-1)(n-2)(n-3)}{2 \cdot 3}$ distinte nel caso del problema studiato da DaRBodx, ciò̀ del problema dei sistemi ortogonali in una varietà, per cui il quadrato dell' elemento lineare è di classe 0 .

$\mathrm{Nel} \S 4$ consideriamo il caso, in cui la equazione algebrica caratteristica ammette delle radici multiple. Allora ad ogni gruppo di $m$ radici eguali ad $\omega_{u}$ corrisponde un gruppo $G_{\imath}$ di $m$ equazioni lineari ed omogenee a derivate parziali di $1 .^{\circ}$ ordine, i cui coefficienti sono determinati a meno di una sostituzione lineare tra di essi, e le condizioni necessarie e sufficienti per la esistenza del sistema di integrali ortogonali cercato consistono in ciò che siano completi: $10^{\circ}$ i sistemi di equazioni che risultano della proposta e di uno dei gruppi $G_{u}$; $2 .^{\circ}$ quelli, che risultano della proposta e di due di questi ultimi gruppi. Allora al gruppo $G_{u}$ di $m$ radici eguali della equazione algebrica caratteristica corrisponde un sistema di $m$ integrali della proposta, i quali possono prendersi come coordinate di una varietà ad $m$ dimensioni contenuta nella data ad $n$. Due qualunque di queste varietà sono ortogonali fra di loro e tutte lo sono alla linea di equazioni:

$$
\frac{d x_{1}}{Y_{1}}=\frac{d x_{2}}{Y_{2}}=\cdots=\frac{d x_{n}}{Y_{n}}
$$

Annali di Matematica, tomo XV. 
Però in questo caso il sistema di integrali ortogonali non è completamente determinato, essendo nell'interno di ciascuna di quelle varietà arbitraria la scelta del sistema di coordinate, purchè ortogonali.

È specialmente notevole e merita forse uno studio speciale il caso, in cui tutte le radici della equazione algebrica caratteristica sono eguali fra di loro. Allora nessuna condizione si richiede per l'esistenza del sistema di integrali ortogonali e questo è affatto indeterminato, poichè ogni integrale della proposta ne può far parte. Quando sia $n=3$ e la varietà, che si considera sia lo spazio ordinario euclideo a tre dimensioni, a questa classe di equazioni appartiene quella, i cui coefficienti sono proporzionali alle derivate del parametro di un sistema di sfere concentriche.

Nel $\S 5$ studieremo il problema dei sistemi $n^{u p l i}$ ortogonali in una varietà qualunque ad $n$ dimensioni e vedremo che in questo caso le equazioni generali del sistema $S_{2}$ sopra accennato prendono delle forme speciali importanti.

$\mathrm{Nel} \S 6$ applicheremo la teoria generale svolta nei precedenti paragrafi al caso di $n=3$. In questo caso, se le due radici della equazione algebrica caratteristica non sono eguali, abbiamo soltanto il sistema di equazioni $S_{1}$, e questo comprende una sola equazione. Perchè invece la equazione algebrica caratteristica abbia le radici eguali debbono essere verificate due equazioni di condizione, le quali sono di $10^{\circ}$ ordine rispetto ai coefficienti $Y_{r}$ ed $a_{r s}$. Malgrado ciò e malgrado che i coefficienti $Y_{r}$ essenzialmente indipendenti si riducano in questo caso precisamente a due, pure ammettendo che la varietà data sia lo spazio euclideo, vedremo che esistono altre equazioni differenziali, per cui le radici della equazione algebrica caratteristica sono eguali fra di loro, oltre a quella ricordata superiormente.

Mi pare in fine utile osservare che la via seguita nel $\S 1$ condurrebbe alla determinazione degli invarianti di un sistema di due forme differenziali ad $n$ variabili, di cui l'una lineare e l'altra quadratica e che anzi le formule ivi trovate conducono immediatamente agli invarianti di $1 .^{\circ}$ ordine. Questa via è quella stessa seguita nella mia Memoria sopra ricordata, e una parte dei risultati, a cui si giungerebbe, coincide con quelli della Memoria stessa, sostituendo nelle formule di questa alle quantità $U^{(r)}$, che rappresentano le derivate di una stessa funzione $U$ rispetto alle variabili $x_{r}$, le funzioni $X_{r}$ qualunque qui considerate, alle $U_{h k}$ le $X_{h k}$, ecc. 


\section{$\S 1$.}

\section{Formule preliminari.}

Due forme differenziali ad $n$ variabili, l'una lineare:

l'altra quadratica:

$$
l=\mathbf{\Sigma}_{r} X_{r} d x_{r}
$$

$$
\varphi^{2}=\mathbf{y}_{r,} a_{r,} d x_{r} d x_{s}
$$

i cui coeffeienti suno funzioni qualunque delle variabili soggette alla sola condizione che il discriminante $a$ della seconda sia $>0$, per la sostituzione di nuove variabili $u$ alle $x$ si trasformano rispettivamente nelle:

essendo:

$$
\begin{aligned}
& (l)=\mathbf{\Sigma}_{p}\left(X_{p}\right) d u_{p} \\
& (\varphi)^{2}=\mathbf{\Sigma}_{p q}\left(a_{p q}\right) d u_{p} d u_{q},
\end{aligned}
$$

$$
\left.\begin{array}{l}
\left(X_{p}\right)=\mathbf{\Sigma}_{r} X_{r} x_{r}^{(p)} \\
\left(a_{p q}\right)=\Sigma_{r s} a_{r s} x_{r}^{(p)} x_{d}^{(q)}
\end{array}\right\}
$$

convenendo di indicare rispettivamente con $x_{r}^{(p)}, x_{r}^{(p q)}$, ecc., $u_{p}^{(r)}, u_{p}^{(r)}$, ecc., le derivate di $x_{r}$ rispetto ad $u_{p}$, ad $u_{p}$ ed $u_{q}$, ecc., e quelle di $u_{p}$ rispetto ad $x_{r}$, ad $x_{r}$ ed $x_{s}$, ecc. In generale converremo di indicare con $U^{(r)}, U^{(r s)}, \ldots$ le derivate rispetto ad $x_{r}$, ad $x_{r}$ ed $x_{s} \ldots$ di una funzione $U$ delle $x$. Converremo di più di distinguere mediante parentesi le quantità relative alle variabili $u$ da quelle analoghe relative alle $x$.

Dalle $(x)$ si hanno mediante derivazione le:

$$
\left.\begin{array}{l}
\left(X_{r}^{(s)}\right)=\Sigma_{h k} X_{h}^{(k)} x_{h}^{(r)} x_{k}^{(s)}+\mathbf{\Sigma}_{h} X_{h} x_{h}^{(r s)} \\
\left.\left(a_{r s, i}\right)=\mathbf{\Sigma}_{g} x_{g}^{(i)} \mid \mathbf{\Sigma}_{h k} a_{h k, g} x_{h}^{(r)} x_{k}^{(s)}+\mathbf{\Sigma}_{h} a_{h g} x_{h}^{(r s)}\right\},
\end{array}\right\}
$$

ponendo:

$$
2 a_{h k, g}=a_{h y}^{(k)}+a_{k g}^{(h)}-a_{h k}^{(g,} .
$$

Se tra le (1) si eliminano le $x_{h}^{(r s)}$, il che si fa risolvendo le seconde di quelle equazioni rispetto alle $x_{k}^{(r s)}$ stesse e sostituendone $\mathrm{i}$ valori cos̀̀ trovati nelle prime, si ottengono le:

$$
\left(X_{p, q}\right)=\Sigma_{r s} X_{r_{2} s} x_{r}^{(p)} x_{s}^{(q)},
$$


posto:

$$
\begin{gathered}
X_{r, s}=X_{r}^{(s)}-\mathbf{\Sigma}_{i} a_{r s, i} Y_{i} \\
Y_{i}=\Sigma_{h} c_{i h} X_{h}, \quad c_{i h}=\frac{d \log a}{d a_{i h h}}
\end{gathered}
$$

colla convenzione (che manterremo in seguito nei casi analoghi) che nella derivazione di $a$ si riguardi $a_{i h}$ come distinto da $a_{h i}$, ed osservando che è:

$$
Y_{h}=\mathbf{\Sigma}_{i}\left(Y_{i}\right) x_{i}^{(h)} \text {. }
$$

Le $(\beta)$ e $(\alpha)$ ci dicono che la forma bilineare $\psi$ di coefficienti $X_{r, s}$ è covariante alle $l$ e $\varphi^{2}$ cos̀ che costruendo tutti gli invarianti algebrici di questo sistema di forme si avrebbero tutti gli invarianti differenziali di $1 .^{\circ}$ ardine del sistema di forme differenziali $l$ e $\varphi^{2}$.

Se in luogo di queste si considerano le reciproche $l^{\prime}$ e $\varphi^{\prime 2}$ di coefficienti $Y_{i}$ e $c_{p q}$, pei quali si hanno invece delle $(\alpha)$ le:

poichè, posto:

$$
\left.\begin{array}{l}
\left(Y_{p}\right)=\mathbf{\Sigma}_{r} Y_{r} u_{p}^{(r)} \\
\left(c_{p q}\right)=\mathbf{\Sigma}_{r s} c_{r s} u_{p}^{(r)} u_{q}^{(s)},
\end{array}\right\}
$$

$$
Y_{r, s}=\mathbf{\Sigma}_{h k} X_{h, k} c_{h r} c_{k s}
$$

per le $(\beta)$ e $\left(\alpha^{b i s}\right)$ si hanno le:

$$
\left(Y_{p q}\right)=\sum_{r s} Y_{r, s} u_{p}^{(r)} u_{q}^{(s)}
$$

si vede che invece della $\psi$ deve considerarsi la forma $\psi^{\prime}$ di coefficienti $Y_{r, s}$, che è covariante a $l^{\prime}$ e $\varphi^{\prime 2}$. Ciò dà luogo ad una specie di dualità potendosi nelle ricerche, che si riferiscono al sistema proposto $l$ e $\varphi^{2}$, introdurre indifferentemente i coefficienti di un sistema o quelli del sistema reciproco ed ottenere così due espressioni reciproche degli stessi risultati.

Dalle (4) si traggono anche le:

$$
\begin{aligned}
& \Sigma_{s} Y_{r, s} a_{k s}=\mathbf{\Sigma}_{h} X_{h, k} c_{h r} \\
& X_{h, k}=\Sigma_{r s} Y_{r, s} a_{h r} a_{k s} .
\end{aligned}
$$

Notiamo ancora che nelle (4) per le $X_{h, k}$ ponendo i loro valori dati dalle (2) ed eseguendo alcune riduzioni si trova:

$$
Y_{r, s}=\mathbf{\Sigma}_{k} c_{k s} Y_{r}^{(k)}-\frac{1}{2} \mathbf{\Sigma}_{h} c_{r s}^{(k)} Y_{k}+\frac{1}{2} \mathbf{\Sigma}_{h k} X_{h}\left(c_{k r} c_{h s}^{(k)}-c_{k s} c_{h r}^{(k)}\right)
$$




\section{$\S 2$.}

\section{Equazione algebrica caratteristica}

di una equazione lineare ed omogenea a derivate parziali di $1 .^{\circ}$ ordine e ad $n$ variabili in una varietà ad $n$ dimensioni.

Assieme alla forma quadratica differenziale considerata nel precedente paragrafo:

$$
\varphi^{2}=\Sigma_{r s} a_{r s} d x_{r} d x_{s}
$$

si consideri la equazione a derivate parziali di $10^{\circ}$ ordine lineare ed omogenea:

$$
\sum_{r} Y_{r} \psi(r)=0
$$

in cui le $Y_{r}$ sono funzioni qualunque di $x_{1}, x_{2}, \ldots x_{n}$ e si cerchi dapprima la condjzione necessaria e sufficiente affinchè ad un integrale $\rho$ della (I) ne corrispondano altri $n-2 \rho_{2}, \rho_{3}, p_{n-1}$ indipendenti fra di loro e tali che si abbia:

$$
\mathbf{\Sigma}_{r s} c_{r s} \rho_{1}^{(r)} \rho_{l}^{(s)}=0 \quad(h=2,3, \ldots n-1),
$$

cioè, come diremo adottando per brevità il linguaggio geometrico, che gli siano ortogonali nello spazio di elemento lineare $\varphi$.

Posto:

$$
\mathrm{H}_{r}=\mathbf{\Sigma}_{s} c_{r s} p^{(s)},
$$

si richiederà per ciò e basterà che la equazione (I) e la:

$$
\mathbf{\Sigma}_{r} \mathrm{H}_{r} \psi^{r)}=0
$$

costituiscano un sistema completo, cioè che indicando con $\Xi_{3}$ ed $\omega$ due indeterminate sia:

ovvero, essendo:

$$
\frac{1}{2} \Sigma_{r}\left(Y_{r} \mathrm{H}_{s}^{(r)}-\mathrm{H}_{r} Y_{s}^{(r)}\right)=\Xi_{s} Y_{s}+\omega \mathrm{H}_{s}
$$

$$
\Sigma_{r} Y_{r} H_{s}^{(r)}=\Sigma_{t} c_{s t} \Sigma_{r} Y_{r} \rho^{(r t)}+\Sigma_{t} \rho^{(t)} \Sigma_{r} Y_{r} c_{s t}^{(r)}=\Sigma_{r} \rho^{(r)} \Sigma_{t}\left(c_{s r}^{(t)} Y_{t}-c_{s t} Y_{r}^{(t)}\right)
$$

che, posto:

$$
2 Y_{r s}=\mathbf{\Sigma}_{t}\left(c_{s t} Y_{r}^{(t)}+c_{r t} Y_{s}^{(t)}-c_{r s}^{(t)} Y_{t}\right)
$$

le $\rho^{(r)}$ soddisfino ad un sistema di equazioni algebriche della forma:

$$
\begin{gathered}
\boldsymbol{\Sigma}_{r} Y_{r \rho^{(r)}=0} \\
Y_{s} \Xi_{0}+\Sigma_{r}\left(Y_{r s}+\omega c_{r s}\right) \rho^{(r)}=0 \quad(s=1,2, \ldots n) .
\end{gathered}
$$


Le quantità $\Xi_{0}$ ed $\omega$ dovranno essere determinate in base a queste stesse equazioni e però $\omega$ dovrà essere radice della equazione algebrica di grado $n-1$ :

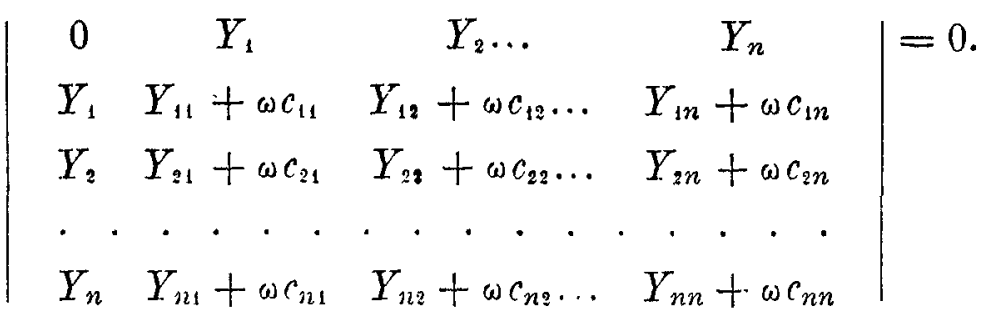

Fermiamoci a studiare questa equazione e dimostriamo che essa ha tutte le radici reali. Se $\omega_{h}$ è una radice della $(e)$ è sempre possibile determinare un sistema di quantità $\Xi_{0 l} \Xi_{1 h} \ldots \Xi_{n h}$, che non siano tutte nulle e che soddisfino alle equazioni:

$$
\left.\begin{array}{c}
\sum_{1}^{n} Y_{r} \Xi_{r h}=0 \\
Y_{s} \Xi_{0 h}+\sum_{1}^{n}\left(Y_{r s}+\omega_{h} c_{r s}\right) \Xi_{r h}=0 .
\end{array}\right\}
$$

Indicando con $\Xi_{0 k}, \Xi_{1 k}, \ldots \Xi_{n k}$ le quantità analoghe corrispondenti ad un'altra radice $\omega_{k}$ della $(e)$ si ha dalle (2) moltiplicate per $\Xi_{s k}$ e sommate rispetto ad $s$ da 1 fino ad $n$

$$
\mathbf{\Sigma}_{r s} Y_{r s} \Xi_{r h} \Xi_{s k}+\omega_{h} \mathbf{\Sigma}_{r s} c_{r s} \Xi_{r h} \Xi_{s h}=0
$$

e sottraendo da questa quella, che si ottiene scambiando $h$ con $k$,

$$
\left(\omega_{h}-\omega_{k}\right) \sum_{r s} c_{r s} \Xi_{r h} \Xi_{s k}=0 .
$$

Supponiamo che $\omega_{h}$ ed $\omega_{k}$ siano radici complesse coniugate della $(e)$. Se è $\Xi_{r h}=A_{r}+i B_{r}$, sarà $\Xi_{s k}=A_{s}-i B_{s}$ e la (3) ci darà :

$$
\left(\omega_{h}-\omega_{k}\right) \sum_{r s} C_{r s}\left(A_{r} A_{s}+B_{r} B_{s}\right)=0,
$$

la quale, se $\omega_{h}$ ed $\omega_{k}$ non sono reali ed eguali, non può essere verificata a meno che sia $\Xi_{r h}=\Xi_{s k}=0$, il che è contro la ipotesi. Possiamo dunque concludere che: La equazione (e) ha tutte le radici reali.

Si dimostra pure con un metodo noto che se una radice $\omega_{h}$ della equazione $(e)$ annulla insieme ad essa tutti i minori principali di ordine $n+1-m$ del determinante, che ne costituisce il primo membro, essa è multipla del grado $m$ e reciprocamente ogni radice multipla del grado $m$ annulla tutti quei minori e per conseguenza anche gli altri minori dello stesso ordine $n+1-m$. In questo caso le equazioni (2) ammettono, come è noto, $m$ e non più di $m$ sistemi indipendenti di soluzioni. 
La equazione (I) mediante la sostituzione di nuove variabili indipendenti $u$ alle $x$ si trasforma nella:

essendo:

$$
\mathbf{\Sigma}_{p}\left(Y_{p}\right)\left(\psi^{(p)}\right)=0
$$

$$
\left(Y_{p}\right)=\Sigma_{r} Y_{r} u_{p^{\prime}}^{(r)} .
$$

Queste confrontate colle prime tra le $\left(\alpha^{b i s}\right)$ del $\$ 1$ ci permettono di prendere per le quantità $Y_{r}$ considerate in quel paragrafo i coefficienti della (I). Confrontando poi ancora le (1) di questo colle (5) del $\S 1$ si vede che è:

$$
Y_{r s}=\frac{1}{2}\left(Y_{r, s}+Y_{s r}\right),
$$

e per quanto si è dimostrato nel paragrafo stesso si conclude che la forma di coefficienti $Y_{r s}$ è covariante a quella di coefficienti $c_{r s}$. Il primo membro della $(e)$ è quindi un controvariante di queste forme e però le sue radici sono indipendenti dalla scelta delle variabili $x_{1}, x_{2}, \ldots x_{n}$. Per questa ragione chiameremo la equazione (e) equazione algebrica caratteristica della equazione differenziale (I) nella varietà di elemento lineare $\varphi$.

Mantenendo sempre le notazioni del $\S 1$ e ponendo:

$$
X_{h k}=\frac{X_{h, h}+X_{k, h}}{2},
$$

cioè:

$$
X_{h k}=\frac{X_{h}^{\left(k^{i}\right.}+X_{k}^{(h)}}{2}-\mathbf{\Sigma}_{i} a_{h k, i} Y_{i}
$$

si hanno dalle (4) di quel paragrafo le:

$$
Y_{r s}=\sum_{h k} X_{h k} c_{h r} c_{k s} .
$$

Queste insieme alle (3) del paragrafo stesso ci dicono che il primo membro della $(e)$ è il prodotto del quadrato del determinante $\mathbf{\Sigma} \pm c_{14} c_{22} \ldots c_{n n}$ per il primo membro della equaz:one:

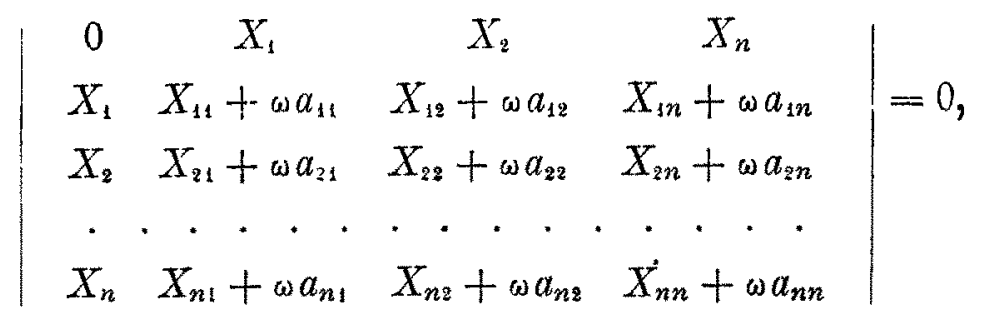

che è quindi la $(e)$ sotto una nuova forma, che potrebbe dirsi reciproca della $(e)$ 
stessa. Le forme $(e)$ ed $\left(e_{1}\right)$ coincidono se è $\varphi^{2}=\sum_{1}^{n} d x_{r}^{2}$. Se in questo caso si suppone di più che sia $\Sigma_{r} Y_{r}^{2}=1$, il che può sempre farsi, e che le $X_{r}=Y_{r}$ siano proporzionali alle derivate di una stessa funzione $\rho$ rispetto alle $x_{r}$ si riconosce subito che la equazione $(e)$ od $\left(e_{1}\right)$ è quella nota pel caso di $n=3$, che ha per radici le curvature principali del sistema di superficie di parametro $p$, mentre per $n$ qualunque si riconosce facilmente che, sempre nelle ipotesi ammesse, se ne può riguardare come la generalizzazione. Se $\varphi^{2}$, pure essendo di classe 0, non è ridotto alla forma $\mathbf{Y}_{r} d x_{r}^{2}$, e le $X_{r}$ sono sempre quantità proporzionali alle derivate di una funzione $\rho$ rispetto alle $x_{r}$ tali che si abbia $\sum_{r} X_{r} Y_{r}=1$, da quanto abbiamo dimostrato possiamo concludere che $(e)$ od $\left(e_{1}\right)$ ci dánno in coordinate generali la equazione, che ha per radici le curvature principali del sistema di superficie di parametro $\rho$.

Notiamo infine che, come si riconosce facilmente, se si moltiplica la equazione proposta (I) per un coefficiente $U$, anche le radici della equazione algebrica caratteristica vengono tutte moltiplicate per lo stesso coefficiente.

\section{$\S 3$.}

Risoluzione del problema dei sistemi di integrali ortogonali di una equazione lineare ed omogenea a derivate parziali di $1 .^{\circ}$ ordine in una varietà, in cui la sua equazione algebrica caratteristica non ha radici multiple.

Proponiamoci in secondo luogo di determinare le condizioni necessarie e sufficienti perchè la equazione (I) ammetta $n-1$ integrali $\rho_{1}, \rho_{2}, \ldots \rho_{n-1}$ ortogonali fra di loro due a due nella varietà di elemento lineare $\varphi$. Se questi integrali esistono ciascuno di essi si trova evidentemente nella condizione dell'integrale $\rho$ considerato nel precedente paragrafo e però soddisfa a un sistema di equazioni della forma (II), in cui per $\omega$ sia posta una radice $\omega_{h}$ della equazione algebrica caratteristica.

Supporremo dapprima che questa non abbia radici multiple. Allora posto:

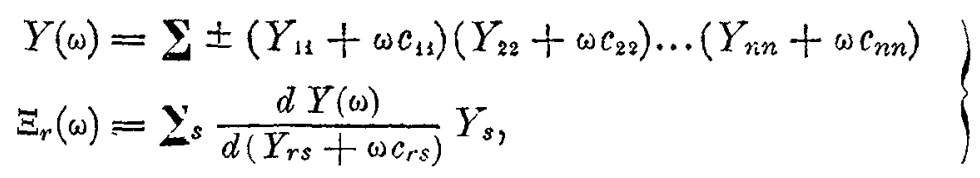

per $\omega=\omega_{h} Y(\omega)$ è differente da 0 e non si annullano insieme tutte le $\Xi_{r}(\omega)$, 
e le equazioni (2) del $\S 2$ ammettono un solo sistema di soluzioni indipendenti, pel quale si puo prendere:

$$
\Xi_{0 h}=-a Y\left(\omega_{h}\right), \quad \Xi_{r h}=a \Xi_{r}\left(\omega_{h}\right) .
$$

Se esiste dunque il sistema di integrali ortogonali $\rho_{1}, \rho_{2}, \ldots p_{n-1}$ ognuno di essi corrisponderà ad una radice della equazione algebrica caratteristica avendosi:

$$
\rho_{h}^{(r)}=\mu_{h} \Xi_{r h},
$$

cioè le $\Xi_{r h}$, qualunque sia $h$, per $r=1,2, \ldots n$ saranno proporzionali alle derivate rispetto alle $x_{r}$ di una funzione $\rho h$. Reciprocamente se le $\Xi_{r h}$, qualunque sia $h$, per $r=1,2, \ldots n$ sono proporzionali alle derivate di una funzione $\rho_{h}$ rispetto alle $x_{r}$ dalla prima delle (2) del $\S 2$ si deduce che $\rho_{1}, \rho_{2}, \ldots$ $\rho_{n-1}$ sono integrali della equazione proposta (I) e dalle (3) dello stesso paragrafo che questi integrali sono ortogonali fra di loro due a due nella varietà di elemento lineare $\varphi$. Possiamo dunque concludere che:

Quando la equazione algebrica caratteristica di una equazione a derivate parziali $d i 1^{\circ}$ ordine lineare ed omogenea ad $n$ variabili $x_{1}, x_{2}, \ldots x_{n}$ in una varietà ad $n$ dimensioni, di cui queste sono le coordinate, non ha radici multiple, le condizioni necessarie e sufficienti perchè questa equazione ammetta un sistema di $n-1$ integrali ortogonali fra di loro due a due nella detta varietà consistono in ciò che, le quantità $\Xi_{r h}$ essendo quelle sopra definite, le equazioni a differenziali totali:

$$
\Sigma_{r} \Xi_{r h} d x_{r}=0 \quad(h=1,2, \ldots n-1),
$$

siano tutte integrabili mediante una sola primitiva $\rho_{h}=$ cost. In tal caso $\rho_{1}$, $\rho_{2}, \ldots \rho_{n-1}$ costituiscono il sistema di inteyrali ortogonali cercati e la integrazione della equazione proposta è ridotta a quella delle $n-1$ equazioni a differenziali totali (III).

Ad illustrazione di questo teorema conviene far vedere come il verificarsi delle condizioni, che secondo il teorema stesso sono necessarie e sufficienti per la esistenza del sistema di integrali ortogonali non dipende dalla scelta delle variabili indipendenti. A ciò si riesce notando ehe per le $\left(\alpha^{b i s}\right)$ e $\left(\beta^{b i s}\right)$ le $Y_{r s}$ essendo coefficienti di una forma covariante a quella di coefficienti $c_{r s}$ reciproca della espressione di $\varphi^{2}$ i coefficienti $a \frac{d Y(\omega)}{d\left(Y_{r s}+\omega c_{r s}\right)}$ saranno invece, qualunque sia $\omega$, coefficienti di una forma covariante a quest'ultima così che si avranno le:

$$
(a)\left(\frac{d Y(\omega)}{d\left(Y_{p q}+\omega c_{p q}\right)}\right)=a \sum_{r s} \frac{d Y(\omega)}{d\left(Y_{r s}+\omega c_{r s}\right)} x_{r}^{(p)} x_{s}^{(q)}
$$


dalle quali, dalle (3') del $\S 2$ nonchè dalle (2) di questo si traggono le:

$$
\left(\Xi_{p h}\right)=\Sigma_{r} \Xi_{r h} x_{r}^{(p)}
$$

che dimostrano appunto ciò, che sopra si è asserito.

Nelle (4) si possono anche comprendere le prime delle $(\alpha)$ del $\S 1$, purche ad $h$ si diano $\mathrm{i}$ valori da 1 fino ad $n$, e si ponga $\Xi_{r n}=X_{r}$. Vediamo cosi che le quantità $\Xi_{r h}$ sono tutte cogredienti fra di loro. Se si pone poi:

$$
\mathrm{H}_{r h}=\mathbf{\Sigma}_{s} c_{r s} \Xi_{s h}
$$

così che per la (3) del $\S 1$ si abbia $H_{r n}=Y_{r}$, si ottengono facilmente le:

$$
\left(\mathrm{H}_{p h}\right)=\sum_{r} \mathrm{H}_{r h} u_{p}^{(r)} \text {, }
$$

che comprendono le prime delle $\left(\alpha^{b i s}\right)$ del $\S 1$ e ci dicono che le $\mathrm{H}_{r h}$ sono tutte cogredienti fra di loro e controgredienti alle $\Xi_{r h}$.

Alle (3) del $\S 2$ si può anche dare la forma:

$$
\sum_{r s} a_{r s} \mathrm{H}_{r h} \mathrm{H}_{s k}=0 \text {, }
$$

le quali valgono per $h$ e $k$ differenti fra loro e scelti fra gli indici $1,2, \ldots n$. Se si usa il linguaggio geometrico queste equazioni ci dicono che le $n$ linee di equazioni:

$$
\frac{d x_{1}}{\mathrm{H}_{1 h}}=\frac{d x_{2}}{\mathrm{H}_{2 h}}=\ldots=\frac{d x_{n}}{\mathrm{H}_{n h}} \quad(h=1,2, \ldots n),
$$

sono ortogonali fra di loro due a due nello spazio di elemento lineare $\varphi$. In linguaggio geometrico si puo quindi dire che le condizioni necessarie e sufficienti per l'esistenza di $n-1$ integrali della (I) ortogonali fra di loro due a due nella varietà di elemento lineare $\varphi$ consistono in ciò che le linee di equazioni (6) per $h=1,2, \ldots n-1$ siano le normali ad $n-1$ sistemi di superficie. Queste condizioni sono suscettibili di espressioni analitiche differenti da quelle, che si dedurrebbero direttamente dal teorema sopra dimostrato applicando i noti criteri del Calcolo Differenziale sulla integrabilità delle equazioni a differenziali totali mediante una sola primitiva ed è della ricerca di queste espressioni che noi dobbiamo ora occuparci.

Per le (5) le equazioni (3) del $\$ 2$ si possono mettere sotto la forma:

$$
\mathbf{\Sigma}_{r} \mathrm{H}_{r h} \Xi_{r k}=0 \text {, }
$$

dalle quali se il sistema di integrali ortogonali esiste si ha per le (3):

$$
\Sigma_{r} H_{r h} \rho_{k}^{(x)}=0,
$$


per $k$ differente da $h$. In questa ipotesi dunque gli integrali $\rho_{1}, \rho_{2}, \ldots \rho_{h-1}$, $\rho_{h+1}, \ldots \rho_{n-1}$ soddisfanno in pari tempo alla proposta (I) e alla:

e quindi anche alla:

$$
\Sigma_{r} \mathrm{H}_{r h} \psi^{(r)}=0 \text {, }
$$

$$
\sum_{r s}\left(Y_{s} \mathbf{H}_{r h}^{(s)}-\mathrm{H}_{s h} Y_{r}^{(s)}\right) \psi^{(r)}=0
$$

che ne è una conseguenza, cos̀̀ che sono identicamente soddisfaite le equazioni:

$$
\mathbf{\Sigma}_{r s}\left(Y_{s} H_{r h}^{(s)}-H_{s h} Y_{r}^{(s)}\right) \Xi_{r k}=0,
$$

per $h$ e $k$ differenti fra loro e presi del resto comunque tra gli indici $1,2, \ldots n-1$.

Sempre nella stessa ipotesi le equazioni $\left(\mathrm{I}_{h}\right)$ ed $\left(\mathrm{I}_{k}\right), k$ essendo differente da $h$, sono soddisfatte da $\psi=\rho i$, purchè $i$ sia differente da $h$ e $k$ cosicchè sono pure identicamente soddisfatte le equazioni:

$$
\Sigma_{r s}\left(\mathrm{H}_{s h} \mathrm{H}_{r k}^{(s)}-\mathrm{H}_{s k} \mathrm{H}_{i h}^{(s)}\right) \Xi_{r i}=0,
$$

$h, k, i$ essendo differenti fra loro ma del resto presi comunque tra gli indici $1,2, \ldots n-1$.

Supponiamo ora che le equazioni $\left(\Omega_{h k}\right)$ ed $\left(\Omega_{h k i}\right)$ siano identicamente soddisfatte. Dalle (7), le quali colle convenzioni fatte sopra, valgono anche per $h$ o $k$ eguale ad $n$, si deduce che il sistema di $n-2$ equazioni algebriche lineari, che si traggono dalla:

$$
\sum_{i}^{n} \Xi_{r k} z_{r}=0
$$

dando a $k$ tutti i valori da 1 ad $n-1$, eccettuato uno speciale valore $h$, ammette i due sistemi di soluzioni indipendenti $z_{r}=Y_{r}, z_{r}=\mathrm{H}_{r h}$ e poichè le $\left(\Omega_{h k}\right)$ ci dicono che esso ammette anche il sistema $z_{r}=\mathbf{\Sigma}_{s}\left(Y_{s} \mathrm{H}_{r h}^{(s)}-\mathrm{H}_{s h} Y_{r}^{(s)}\right)$, questo dovrà essere funzione lineare di quelli, cioè dovrà aversi:

$$
\Sigma_{s}\left(Y_{s} \mathrm{H}_{r h}^{(s)}-\mathrm{H}_{s h} Y_{r}^{(s)}\right)=\lambda_{h} Y_{r}+\mu_{h} \mathrm{H}_{r h},
$$

$\lambda_{h}$ e $\mu_{h}$ essendo coefficienti, che sarebbe facile determinare. Analogamente dalle $\left(\Omega_{h h i}\right)$ si traggono le:

$$
\sum_{s}\left(\mathrm{H}_{s h} \mathrm{H}_{r k}^{(s)}-\mathrm{H}_{s h} \mathrm{H}_{r h}^{(s)}\right)=\lambda_{h k} Y_{r}+\mu_{h k} \mathrm{H}_{r h}-\mu_{k h} \mathrm{H}_{r k} .
$$

Le equazioni (8) e (9) ci dicono che il sistema di $n-1$ equazioni lineari ed omogenee a derivate parziali di $10^{\circ}$ ordine, il quale risulta della (I) e di tutte le $\left(\mathrm{I}_{h}\right)$ eccettuata quella, che corrisponde ad un determinato valore $i$ di $h$, è completo ed ammette quindi un integrale $\rho_{i}$, per cui si arrà:

$$
\Sigma_{r} \mathrm{H}_{r h \rho_{i}^{(r)}}=0
$$


per $h=1,2, \ldots i-1, i+1, \ldots n$. E poichè le $(7)$ ci dicono che per gli stessi valori di $h$ il sistema di $n-1$ equazioni algebriche:

$$
\sum_{1}^{n} \mathrm{H}_{r h} z_{r}=0
$$

è soddisfatto anche da $z_{r}=\Xi_{r i}$ converrà concludere che è:

$$
\rho_{i}^{(r)}=\mu_{i} \Xi_{r i}
$$

e quindi per il teorema dimostrato in questo paragrafo che la equazione (I) ammette un sistema di $n-1$ integrali ortogonali nella varietà di elemento lineare $\varphi, i$ quali sono precisamente $\rho_{1}, \rho_{2}, \ldots \rho_{n-1}$.

Possiamo dunque concludere che:

Le condizioni necessarie e sufficienti perchè nel caso, che consideriamo, esista un sistema di $n-1$ integrali della (I) ortogonali fra di loro due a due nella varietà di elemento lineare $\varphi$ analiticamente si traducono nell'essere soddisfatte identicamente le equazioni $\left(\Omega_{h k}\right)$ ed $\left(\Omega_{h k i}\right)$.

$\mathrm{Ci}$ occuperemo ora successivamente di trasformare questi sistemi di equazioni.

Le (2') e (3) del $\S 2$ combinate dánno le:

$$
\boldsymbol{\Sigma}_{r s} Y_{r s} \Xi_{r h} \Xi_{s k}=0
$$

le quali per le (5) dello stesso paragrafo e per le (5) di questo si possono anche mettere sotto la forma:

$$
\Sigma_{r s} X_{r s} \mathrm{H}_{r h} \mathrm{H}_{s k}=0 \text {, }
$$

e che valgono per $h$ e $k$ differenti fra loro presi tra gli indici $1,2, \ldots n-1$.

Le seconde tra le (2) del $\S 2$ si possono poi per le (5) di questo mettere sotto la forma:

$$
\omega_{h} \mathrm{H}_{s h}=-\Xi_{0 h} Y_{s}-\Sigma_{r} Y_{r s} \Xi_{r h} .
$$

Ciò premesso notiamo che per le (5) si ha:

$$
\mathbf{\Sigma}_{s} \mathrm{H}_{s h} Y_{r}^{(s)}=\mathbf{\Sigma}_{p} \Xi_{p h} \mathbf{\Sigma}_{s} c_{s p} Y_{r}^{(s)}
$$

e per le (4) del $\S 2$ e le (11) di questo:

$$
\begin{gathered}
\mathbf{\Sigma}_{s} \mathrm{H}_{s h} Y_{r}^{(s)}=\mathbf{\Sigma}_{p} \Xi_{p h} Y_{p r}-\mathbf{\Sigma}_{p} \Xi_{p h} \mathbf{\Sigma}_{s} c_{r s} Y_{p}^{(s)}+\mathbf{\Sigma}_{p} \Xi_{p h} \mathbf{\Sigma}_{s} c_{p r}^{(s)} Y_{s}= \\
=-\omega_{h} \mathrm{H}_{r h}-\Xi_{0 h} Y_{r}-\mathbf{\Sigma}_{p} \Xi_{p h} \mathbf{\Sigma}_{s} c_{s r} Y_{p}^{(s)}+\mathbf{\Sigma}_{p} \Xi_{p h} \mathbf{\Sigma}_{s} c_{p r}^{(s)} Y_{s},
\end{gathered}
$$

e da queste tenendo conto delle (7):

$$
\mathbf{\Sigma}_{r s} Y_{r}^{(s)} H_{s h} \Xi_{r k}=-\mathbf{\Sigma}_{r s} Y_{r}^{(s)} \Xi_{r h} \mathrm{H}_{s k}+\mathbf{\Sigma}_{p} Y_{p} \mathbf{\Sigma}_{r s} c_{r s}^{(p)} \Xi_{r h} \Xi_{s k} .
$$


Per le (3) del $\S 2$ essendo:

$$
\boldsymbol{\Sigma}_{r s} c_{r s}^{(p)} \Xi_{r h} \Xi_{s h}=-\boldsymbol{\Sigma}_{r} \mathrm{H}_{r h} \Xi_{i k}^{(p)}-\boldsymbol{\Sigma}_{r} \mathrm{H}_{r k} \Xi_{i h}^{(p)}
$$

abbiamo ancora:

$$
\mathbf{\Sigma}_{r s} Y_{r}^{(s)} \mathrm{H}_{s l} \Xi_{r h}=-\mathbf{\Sigma}_{r s} Y_{r}^{(s)} \Xi_{r h} \mathrm{H}_{s k}-\mathbf{\Sigma}_{r s} Y_{s}\left(\mathrm{H}_{r h} \Xi_{: k}^{(s)}+\mathrm{H}_{r k} \Xi_{r h}^{(s)}\right)
$$

$e$ in fine, tenendo conto ancora delle (7):

$$
\mathbf{\Sigma}_{r s}\left(Y_{s} \mathrm{H}_{r h}^{(s)}-\mathrm{H}_{s h} Y_{r}^{(s)}\right) \Xi_{r h}=-\mathbf{Z}_{r s}\left(Y_{s} \mathrm{H}_{r h}^{(s)}-\mathrm{H}_{s h} Y_{r}^{(s)}\right) \Xi_{r l} .
$$

Dalla (7) si trae pure facilmente la:

$$
\mathbf{\Sigma}_{r s}\left(Y_{s} \mathrm{H}_{r h}^{(s)}-\mathrm{H}_{s h} Y_{r}^{(s)}\right) \Xi_{r h}=\mathbf{\Sigma}_{r s} Y_{r} \mathrm{H}_{s h}\left(\Xi_{r k}^{(s)}-\Xi_{d k}^{(r)}\right)
$$

Ma per le (13) si hanno le:

$$
\begin{gathered}
\omega_{h} \Sigma_{r s} Y_{r} \mathrm{H}_{s h}\left(\Xi_{r h}^{(s)}-\Xi_{s k}^{r)}\right)=-\Sigma_{q r s} Y_{r} Y_{q s} \Xi_{q h}\left(\Xi_{r k}^{(s)}-\Xi_{r k}^{(r)}\right)= \\
=\Sigma_{q r s} Y_{q s} Y_{r}^{(s)} \Xi_{q h} \Xi_{r h}+\Sigma_{q} Y_{q} \Sigma_{r s} Y_{r s} \Xi_{r h} \Xi_{s k}^{(q)}
\end{gathered}
$$

dalle quali facendo uso delle (10) derivate rispetto ad $x_{q}$ e poi di nuovo delle (11), cambiatovi $h$ in $k$, e dalle (12) si traggono le:

$$
\left(\omega_{h}-\omega_{h}\right) \Sigma_{r s} Y_{r} \mathrm{H}_{s h}\left(\Xi_{r h}^{(s)}-\Xi_{s k}^{(r)}\right)=\Sigma_{r s} Y_{r s}^{\prime} \Xi_{r h} \Xi_{s h}
$$

posto:

$$
2 Y_{r s}^{\prime}=\Sigma_{i}\left(Y_{i s} Y_{r}^{(i)}+Y_{i r} Y_{s}^{(i)}-Y_{r s}^{(i)} Y_{i}\right) .
$$

Per le (13) e (14) alle equazioni $\left(\Omega_{h k}\right)$ pud dunque darsi la forma:

$$
\Sigma_{r s} Y_{r s}^{\prime} \Xi_{r h} \Xi_{s k}=0
$$

in cui $h$ e $k$ sono due indici differenti scelti tra i numeri $1,2, \ldots n-1$. 亡 da notare che i coefficienti $Y_{r s}^{\prime}$ si compongono coi coefficienti $Y_{r}$ ed $Y_{r s}$, come questi ultimi coi coefficienti $Y_{r}$ e $c_{r s}$ e poichè, come risulta dal $\S 1$, gli $Y_{r s}$ sono coefficienti di una forma covariante a quella di coefficienti $c_{r s}$, lo stesso sarà degli $Y_{r s}^{\prime}$ cioè sarà :

$$
\left(Y_{p q}^{\prime}\right)=\Sigma_{r s} Y_{r s}^{\prime} u_{p}^{(r)} u_{q}^{(s)}
$$

Queste combinate colle (4) ci dicono che le espressioni, che si trovano al primo membro delle $\left(\Omega^{\prime}{ }_{h k}\right)$, sono invariabili.

Alle $\left(\Omega_{h k}\right)$ può darsi ancora un'altra forma, che potrebbe dirsi reciproca della $\left(\Omega_{h k}^{\prime}\right)$, perchè è analoga a questa, ma contiene invece delle quantità $\Xi_{r h}$ le controvarianti $H_{r h}$. Per far ciò basta nelle $\left(\Omega_{h k}^{\prime}\right)$, sostituire alle $\Xi_{r h}$ i loro 
valori tolti dalle (5). Esse si cambiano allora nella:

essendo:

$$
\mathbf{\Sigma}_{r s} X_{r s}^{\prime} \mathrm{H}_{1 \cdot h} \mathrm{H}_{s k}=0
$$

$$
X_{r s}^{\prime}=\mathbf{\Sigma}_{p q} a_{p r} a_{q s} Y_{p q}^{\prime} \text {. }
$$

Anche le espressioni, che si trovano ai primi membri delle $\left(\Omega^{\prime \prime}{ }_{h k}\right)$ sono invariabili e se si fa uso delle (1) e (5) del $\S 2$ si trovano dopo alcune riduzioni per i coefficienti $X_{r s}^{\prime}$ le espressioni:

$$
X_{r_{s}}^{\prime}=2 \mathbf{\Sigma}_{p q} c_{p q} X_{p r} X_{q s}-\frac{1}{2} \mathbf{\Sigma}_{p}\left(X_{p r} Y_{p}^{(s)}+X_{p s} Y_{p}^{(r)}+X_{r s}^{(p)} Y_{p}\right)
$$

Le equazioni $\left(\Omega_{h k}\right)$, come si deduce dalle (12), sono in numero di $\frac{(n-1)(n-2)}{2}$ distinte e in questo numero appaiono appunto quando sono messe sotto le forme $\left(\Omega^{\prime}{ }_{h k}\right)$ od $\left(\Omega^{\prime \prime}{ }_{h k}\right)$.

Passando ora alle equazioni $\left(\Omega_{h k i}\right)$ comincieremo dal notare che dalle (7) si ha:

$$
\Sigma_{r s}\left(\mathrm{H}_{s h} \mathrm{H}_{r k}^{(s)}-\mathrm{H}_{s k} \mathrm{H}_{r h}^{(s)}\right) \Xi_{r i}=\sum_{r s} \mathrm{H}_{r h} \mathrm{H}_{s k}\left(\Xi_{r i}^{(s)}-\Xi_{s i}^{(r)}\right) .
$$

Notiamo ancora che dalle (5) e (1) si ha per un noto teorema sui determinanti:

$$
\mathrm{H}_{r \cdot h}=\mathbf{\Sigma}_{k} Y_{k} \mathbf{\Sigma}_{s} \frac{d a}{d a_{s r}} \frac{d Y\left(\omega_{h}\right)}{d\left(Y_{s k}+\omega_{h} c_{s k}\right)}=\mathbf{\Sigma}_{k} Y_{k} \delta_{r k}
$$

indicando con $\delta_{r k}$ il complemento algebrico di $d_{r k}$ nel determinante, i cui elementi sono

$$
d_{r k}=\Sigma_{l} a_{r l} Y_{k l}+\varepsilon \omega_{h},
$$

essendo $\varepsilon=1$ per $r=k, \varepsilon=0$ in ogni altro caso. Si hanno dunque anche per le (3) del $\S 1$ e applicando di nuovo l'accennato teorema le:

$$
\mathrm{H}_{r_{i}}==\frac{1}{a} \boldsymbol{\Sigma}_{i} X_{i} \boldsymbol{\Sigma}_{k} \frac{d a}{d a_{i k}} \delta_{r k}=\frac{1}{a} \boldsymbol{\Sigma}_{i} \mathrm{X}_{i} \frac{d X\left(\omega_{h}\right)}{d\left(X_{i r}+\omega_{h} a_{i r}\right)}
$$

posto:

$$
X(\omega)=\mathbf{\Sigma} \pm\left(X_{11}+\omega a_{11}\right)\left(X_{22}+\omega a_{22}\right) \cdots\left(X_{n n}+\omega a_{n n}\right) .
$$

Se si pone ancora:

le $(19)$ e la $\left(e_{1}\right)$ ci dàrno le:

$$
\mathrm{H}_{0 h}=-\frac{1}{a} X\left(\omega_{h}\right)
$$

$$
\left.\begin{array}{c}
\Sigma_{r} X_{r} \mathrm{H}_{r h}=0 \\
\omega_{h} \Xi_{s h}=-\mathrm{H}_{0 h} X_{s}-\mathbf{\Sigma}_{r} X_{r s} \mathrm{H}_{r h},
\end{array}\right\}
$$

le quali non sono che le (11) poste sotto forma reciproca. 
Deriviamo ora una qualunque delle $\left(10^{\prime}\right)$ rispetto ad $x_{q}$, moltiplichiamola poi per $\mathrm{H}_{q i}$, e sommiamo rispetto a $q$ da 1 ad $n$. Si trova così:

$$
-\Sigma_{r s q} X_{r 3}^{(q)} \mathrm{H}_{r h} \mathrm{H}_{s k} \mathrm{H}_{q i}=\Sigma_{r s q}\left(X_{r s} \mathrm{H}_{s k} \mathrm{H}_{q i} \mathrm{H}_{r h}^{(q)}+X_{r s} \mathrm{H}_{r h} \mathrm{H}_{q i} \mathrm{H}_{s k}^{(q)}\right),
$$

e nel secondo membro per $\boldsymbol{\Sigma}_{s} X_{r s} \mathrm{H}_{s k}$ e $\mathbf{\Sigma}_{r} X_{r s} \mathrm{H}_{r h}$ ponendo i valori dati dalle $\left(11^{\prime}\right)$ :

$$
\begin{aligned}
-\sum_{r q q} X_{r s}^{(q)} \mathrm{H}_{r h} \mathrm{H}_{s k} \mathrm{H}_{q i} & =\omega_{k} \Sigma_{r q} \Xi_{r k}^{(q)} \mathrm{H}_{q i} \mathrm{H}_{r h}+\omega_{h} \sum_{r q} \Xi_{r h}^{(q)} \mathrm{H}_{q i} \mathrm{H}_{r k} \\
& +\mathrm{H}_{0 k} \sum_{r q} X_{r}^{(q)} \mathrm{H}_{q i} \mathrm{H}_{r h}+\mathrm{H}_{0 h} \sum_{r q} X_{r}^{(q)} \mathrm{H}_{q i} \mathrm{H}_{r \cdot k},
\end{aligned}
$$

e da questa sottraendo quelle, che se ne ottengono scambiando successivamente $i$ con $h$ e $k$ :

$$
\left.\begin{array}{c}
\Sigma_{r s q}\left(X_{q r}^{(s)}+X_{q s}^{(r)}-X_{r s}^{(q)}\right) \mathrm{H}_{r h} \mathrm{H}_{s k} \mathrm{H}_{q i}=\omega_{h} \mathbf{\Sigma}_{r q} \mathrm{H}_{q i} \mathrm{H}_{r h}\left(\Xi_{r h}^{(q)}-\Xi_{q h}^{(r)}\right) \\
+\omega_{k} \mathbf{\Sigma}_{r q} \mathrm{H}_{q i} \mathrm{H}_{r h}\left(\Xi_{r k}^{(q)}-\Xi_{q k}^{(r)}\right)-\omega_{i} \mathbf{\Sigma}_{q r} \mathrm{H}_{q h} \mathrm{H}_{r k}\left(\Xi_{r i}^{(q)}+\Xi_{q i}^{(r)}\right) \\
+\mathrm{H}_{0 h} \mathbf{\Sigma}_{r q} \mathrm{H}_{q i} \mathrm{H}_{r k}\left(X_{r}^{(q)}-X_{q}^{(1)}\right)+\mathrm{H}_{o h} \mathbf{\Sigma}_{r q} \mathrm{H}_{q i} \mathrm{H}_{r h}\left(X_{r}^{(q)}-X_{q}^{(r)}\right) \\
-\mathrm{H}_{o i} \mathbf{\Sigma}_{r q} \mathrm{H}_{r h} \mathrm{H}_{q h}\left(X_{r}^{(q)}+X_{q}^{(r)}\right) .
\end{array}\right\}
$$

Osservando poi che facendo uso delle $\Xi_{q i}=\Sigma_{s} a_{s q} \mathrm{H}_{s i}$ tratte dalle (5) si trova:

$$
\begin{aligned}
\Sigma_{q r} \mathrm{H}_{q h} \mathrm{H}_{r k}\left(\Xi_{q i}^{(r)}+\Xi_{r i}^{(q)}\right) & =2 \sum_{q r s} a_{q r, s} \mathrm{H}_{s i} \mathrm{H}_{q h} \mathrm{H}_{r k}+\Sigma_{q r s} a_{q r}^{(s)} \mathrm{H}_{s i} \mathrm{H}_{q h} \mathrm{H}_{r k} \\
& -\sum_{r s s} \mathrm{H}_{s i}\left(\mathrm{H}_{r k} \Xi_{s h}^{(r)}+\mathrm{H}_{r h} \Xi_{s k}^{(r)}\right),
\end{aligned}
$$

ovvero, avendosi dalle (7):

$$
\begin{aligned}
& \mathbf{\Sigma}_{q_{r}} a_{r q}^{(s)} \mathrm{H}_{q h} \mathrm{H}_{r k}=\mathbf{\Sigma}_{q}\left(\mathrm{H}_{q h} \Xi_{q k}^{(s)}+\mathrm{H}_{q^{k}} \mathrm{H}_{q k}^{(s)}\right), \\
& \Sigma_{q r} \mathrm{H}_{q h} \mathrm{H}_{r k}\left(\Xi_{q^{i}}^{(r)}+\Xi_{r^{2}}^{(q)}\right)=2 \mathbf{\Sigma}_{q \cdot s} \alpha_{r q, s} \mathrm{H}_{r k} \mathrm{H}_{s i} \mathrm{H}_{q h}+\sum_{q r} \mathrm{H}_{q h} \mathrm{H}_{r i}\left(\Xi_{q k}^{(r)}-\Xi_{r \cdot k}^{(q)}\right) \\
& +\Sigma_{q r} \mathrm{H}_{q k} \mathrm{H}_{r i}\left(\Xi_{q h}^{(r)}-\Xi_{r h}^{(q)}\right)
\end{aligned}
$$

e che quindi per le (11') essendo:

si ha:

$$
\omega_{i} \mathrm{H}_{s i}=\omega_{i} \Sigma_{p} c_{p s} \mathrm{H}_{p i}=-\mathrm{H}_{0 i} Y_{s}-\mathbf{\Sigma}_{p t} c_{p s} X_{p t} \mathrm{H}_{t i}
$$

$$
\begin{gathered}
\omega_{i} \Sigma_{q r} \mathrm{H}_{q h} \mathrm{H}_{r k}\left(\Xi_{q^{2}}^{(r)}+\Xi_{r i}^{(q)}\right)=-2 \mathrm{H}_{o i} \Sigma_{q r s} a_{2 \cdot s, q} Y_{s} \mathrm{H}_{q h} \mathrm{H}_{r k} \\
-2 \Sigma_{p q r s t} c_{p t} a_{r s, t} X_{p q} \mathrm{H}_{r h} \mathrm{H}_{s k} \mathrm{H}_{q i}+ \\
+\omega_{i} \Sigma_{q r}\left\{\mathrm{H}_{q i} \mathrm{H}_{r h}\left(\Xi_{r k}^{(q)}-\Xi_{q k}^{(r)}\right)+\mathrm{H}_{q i} \mathrm{H}_{r k}\left(\Xi_{r h}^{(q)}-\Xi_{q h}^{(r)}\right)\right\}
\end{gathered}
$$

tenuto conto anche delle $\left(10^{\prime}\right)$ e posto:

$$
X_{r s, q}=X_{r q}^{(s)}+X_{s q}^{(r)}-X_{r s}^{(q)}-2 \Sigma_{p t} c_{p t} a_{r s, t} X_{p q}
$$


si può dare alle (20) la forma:

$$
\begin{gathered}
\left(\omega_{k}-\omega_{i}\right) \Sigma_{q r} \mathrm{H}_{q i} \mathrm{H}_{r h}\left(\Xi_{r k}^{(q)}-\Xi_{q k}^{(r)}\right)+\left(\omega_{h}-\omega_{i}\right) \Sigma_{q r} \mathrm{H}_{q i} \mathrm{H}_{r k}\left(\Xi_{r h}^{(q)}-\Xi_{q h}^{(r)}\right)= \\
\boldsymbol{\Sigma}_{r s q} X_{r s, q} \mathrm{H}_{r h} \mathrm{H}_{s k} \mathrm{H}_{q i}+\mathbf{\Sigma}_{q r}\left(\mathrm{H}_{0 h} \mathrm{H}_{r k}+\mathrm{H}_{0 k} \mathrm{H}_{r h}\right) \mathrm{H}_{q i}\left(X_{q}^{(r)}-X_{r}^{(q)}\right) .
\end{gathered}
$$

Da queste e dalle (18) è facile concludere che il sistema di equazioni $\left(\Omega_{h k i}\right)$ equivale al sistema:

$$
\sum_{r s q} X_{r s, q} \mathrm{H}_{r h} \mathrm{H}_{s k} \mathrm{H}_{q i}+\mathbf{\Sigma}_{q r}\left(\mathrm{H}_{0 h} \mathrm{H}_{r k}+\mathrm{H}_{o k} \mathrm{H}_{r h}\right) \mathrm{H}_{q i}\left(X_{q}^{(\cdot)}-X_{r}^{(q)}\right)=0 . \quad\left(\Omega^{\prime}{ }_{h k i}\right)
$$

Il loro numero è $\frac{(n-1)(n-2)(n-3)}{2}$.

Sarebbe facile dimostrare che le espressioni:

$$
\Sigma_{r s q} X_{r s, q} \mathrm{H}_{r h} \mathrm{H}_{s k} \mathrm{H}_{q i}, \quad \boldsymbol{\Sigma}_{r q}\left(X_{q}^{(r)}-X_{r}^{(q)}\right) \mathrm{H}_{r k} \mathrm{H}_{q i},
$$

le quali si trovano al primo membro delle $\left(\Omega^{\prime}{ }_{h k i}\right)$, sono invariabili, come pure il dare a queste la forma reciproca, ma su ciò non ci tratterremo.

\section{$\S 4$.}

\section{Risoluzione del problema dei sistemi di integrali ortogonali nel caso generale.}

Prima di passare a trattare del problema propostoci nel paragrafo precedente nel caso più generale, occupiamoci ancora di un caso speciale, cioè di quello, in cui tutte le radici della equazione algebrica caratteristica sono eguali fra di loro. In tal caso, se per $\omega_{h}$ si pone il valor comune di queste radici, le equazioni (2) del $\S 2$ si riducono a due soltanto indipendenti fra di loro e, non tenendo conto della prima, la quale è sempre soddisfatta per $\Xi_{r h}=\rho_{h}^{(r)}$, se $\rho_{h}$ è un integrale della (I), ad una sola, la quale serve a determinare la $\Xi_{0 h}$ lasciando arbitrarie le $\Xi_{r h}(r=1,2, \ldots n)$. In questo caso dunque secondo il teorema del $\S 2$ per ogni integrale $\rho_{1}$ della (I) ne esistono altri $n-2$, che gli sono ortogonali nella varietà di elemento lineare $\varphi$. Siano questi $\rho_{2}, \rho_{3}, \ldots$ $\rho_{n-1}$. Esisterà un sistema di $n-2$ integrali $\chi_{1}, \chi_{3}, \ldots \chi_{n-1}$ ortogonali a $\rho_{2}$ nella varietà di elemento lineare $\varphi$, per cui cioè sarà:

$$
\mathbf{\Sigma}_{r s} c_{r s} \rho_{2}^{(r)} \chi_{h}^{(s)}=0 \quad(h=1,3,4, \ldots n-1),
$$


e avendosi:

$$
\begin{gathered}
\chi_{h}^{(s)}=\sum_{1}^{n-1} \frac{d \chi_{h}}{d \rho_{q}} \rho_{q}^{(s)} \\
\sum_{1}^{n-1} \frac{d \eta_{h}}{d p_{q}} \mathbf{\Sigma}_{r s} c_{r s} \rho_{2}^{(r)} \rho_{q}^{(s)}=0 .
\end{gathered}
$$

Poichè è $\boldsymbol{\Sigma}_{r s} c_{r s} \rho_{2}^{(r)} \rho_{1}^{(s)}$

$$
\sum_{2}^{n-1} \frac{d y_{h}}{d \rho_{q}} \sum_{r s} c_{r s} \rho_{2}^{(r)} \rho_{\Psi}^{(s)}=0 \quad(h==1,3, \ldots n-1) .
$$

Se ora invece di $\chi_{1}, \chi_{3}, \ldots \chi_{n-1}$ prendiamo $\rho_{1}, \chi_{3}^{\prime}, \chi_{4}^{\prime}, \ldots \chi_{n-1}^{\prime}$, con $\chi_{3}^{\prime}$ indicando cio, che diviene' $\chi_{h}$ ponendovi per $\rho_{1}$ una costante, noi abbiamo per la precedente:

$$
\Sigma_{r s} \rho_{r s} \rho_{2}^{(r)} \chi_{h}^{\prime(s)}=\sum_{2}^{n-1} q \frac{d \chi_{h}}{d \rho_{q}} \sum_{r s} c_{r s} \rho_{2}^{(r)} \rho_{\Psi}^{(\prime)}=0 \quad(h=3,4, \ldots n-1) .
$$

Da queste e dall'essere $\mathbf{\Sigma}_{r s} c_{r s} \rho_{3}^{(r)} p_{1}^{(s)}=0$, concludiamo che se in luogo delle $\rho_{3}, \rho_{4}, \ldots \rho_{n-1}$, che si avevano, si prendono le $\chi_{3}^{\prime}, \chi_{4}^{\prime}, \ldots \chi_{n-1}^{\prime}$, che ne sono funzioni, seguitando però a denotarle come prima, il nuovo sistema $\rho_{1}, \rho_{2}, \ldots$ $\rho_{n-1}$ è tale che non soltanto $\rho_{1}$ e $\rho_{2}$ sono ortogonali fra di loro, ma $\rho_{3}, \rho_{4}, \ldots \rho_{n-1}$ lo sono ad amendue. Si dimostra egualmente come si possano scegliere le $\rho_{4}$, $\rho_{3}, \ldots \rho_{n-1}$ in modo che siano ortogonali non soltanto a $\rho_{1}$ e $\rho_{2}$, ma anche a $\rho_{3}$ e proseguendo cosi si conclude che:

Quando la equazione algebrica caratteristica di una equazione lineare ed omogenea a derivate parziali di $10^{\circ}$ ordine in ura varietà ad $n$ dimensioni ha tutte le radici eguali, per ogni suo integrale se ne possono sempre ed in infiniti modi scegliere altri $n-2$, che con essa costituiscano un sistema di integrali ortogonali in quella varietà.

Nel caso più generale le radrei della equazione algebrica caratteristica si dividono in tanti gruppi $G_{1}, G_{2}, \ldots$ il gruppo $G_{h}$ in generale contenendo tutte le radici, che hanno uno stesso valore $\omega_{h}$ e il cui numero indicheremo con $m_{h}$. Allora il sistema di equazioni (2) del $\S 2$ ammette $m_{h}$ sistemi di soluzioni indipendenti, che indicheremo con $\Xi_{r h_{1}}, \Xi_{r h_{2}}, \ldots$ e se esiste il sistema di $n-1$ integrali della (I) ortogonali nella varietà di elemento lineare $\varphi$, le derivate rispetto alle $x_{r}$ di uno qualunque di essi dovendo soddisfare ad un sistema di equazioni (2), nel quale $\omega_{h}$ sia una radice della equazione algebrica caratteristica, gli integrali stessi si divideranno in tanti gruppi corrispondenti ai gruppi 
$G_{h}$ delle radici di questa e contenenti rispettivamente $m_{h}$ integrali. Posto ora:

$$
\mathrm{H}_{r h_{i}}=\mathbf{\Sigma}_{s} c_{r s} \Xi_{s h_{i}} \quad\left(i=1,2, \ldots m_{h}, \quad r=1,2, \ldots n\right),
$$

consideriamo il sistema di $m_{h}$ equazioni:

$$
\mathbf{\Sigma}_{r} \mathrm{H}_{r} h_{i} \psi^{r)}=0, \quad\left(i=1,2, \ldots m_{l}\right)
$$

o sia $\rho_{k}$ un integrale del sistema ortogonale corrispondente ad un gruppo $G_{k}$ differente da $G_{h}$. Per quanto si è detto le $\rho_{k}^{(r)}$ soddisfaranno al sistema di equazioni (2) del $\S 2$, in cui sia cambiato $h$ in $k$ e per le (3) dello stesso paragrafo soddisfaranno anche alle equazioni $\left(\mathrm{I}_{h}\right)$. Il sistema di $m_{h}+1$ equazioni, che risulta della $(\mathrm{I})$ e delle $\left(\mathrm{I}_{h}\right)$ ammette dunque $n-m_{h}-1$ integrali indipendenti e quindi è completo. In egual modo si vede che è completo il sistema di equazioni, che risulta della $(\mathrm{I})$ e di due gruppi $\left(\mathrm{I}_{h}\right),\left(\mathrm{I}_{k}\right)$ corrispondenti a due radici non eguali $\omega_{, 2}$ ed $\omega_{k}$ della equazione algebrica caratteristica.

Reciprocamente supponiamo che siano completi tutti i sistemi, che risultano della (I) e di uno qualunque dei sistemi $\left(\mathrm{I}_{h}\right)$ e cosi pure quelli, che risultano della (I) e di due sistemi $\left(\mathrm{I}_{h}\right),\left(\mathrm{I}_{k}\right)$. Sarà allora completo anche jl sistema di $n-m_{h}$ equazioni, che risulta della proposta e di tutti i gruppi $\left(\mathrm{I}_{k}\right)$, eccettuato uno speciale $\left(\mathrm{I}_{h}\right)$. Se indichiamo con $\rho_{i_{i}}\left(i=1,2, \ldots m_{h}\right)$ gli integrali di questo sistema di equazioni, le $\rho_{h_{i}}^{r \gamma}$ soddisfanno al sistema di $n-m_{h}$ equazioni algebriche lineari ed omogenee ad $n$ incognite, che risultano della:

e dei gruppi della forma:

$$
\mathbf{\Sigma}_{r} Y_{r} l_{r}=0
$$

$$
\mathbf{\Sigma}_{r} \mathrm{H}_{r k_{i}} l_{r}=0 \quad\left(i=1,2, \ldots m_{k}\right)
$$

con esclusione di quello, che corrisponde alla radice $\omega_{h}$. Per la prima delle (2) e per le (3) del $\S 2$ alle stesse equazioni soddisfanno anche gli $m_{h}$ sistemi $\Xi_{h_{\iota}}\left(i=1,2, \ldots m_{h}\right)$ indipendenti fra di loro, dal che si deduce che le $\rho_{h_{i}}^{(2)}$ sono funzioni lineari delle $\Xi_{r h}$ e quindi soddisfanno alle (2) del $\S 2$ poste invece delle $\Xi_{, h}(r=1,2, \ldots n)$, purchè a $\Xi_{0 h}$ si dia un conveniente valore. Abbiamo così per ogni gruppo $G_{h}$ di $m_{h}$ radici eguali ad $\omega_{h}$ della equazione algebrica caratteristica dimostrata la esistenza di un gruppo $g_{h}$ di $m_{h}$ integrali $\rho_{h_{i}}\left(i=1,2, \ldots m_{i}\right)$ tali che le $\rho_{h_{i}}^{(r)}$ soddisfanno alle equazioni (2) del $\S 2$. Le (3) dello stesso paragrafo dimostrano che due integrali $\rho_{h}, \rho_{h}$ di due differenti gruppi $g_{h}, g_{k}$ sono ortogonali fra di loro nella varietà di elemento lineare $\varphi$. Però pel teorema dimostrato in quel paragrafo ognuno di questi integrali 
della (I), per esempio $\rho_{h_{1}}$, ne ammette altri $n-2$, che gli sono ortogonali ed è facile vedere come per questi si possano prendere gli $n-m_{h}-1$ integrali dei gruppi $g_{k}$ corrispondenti a gruppi di radici eguali, diversi da $G_{h}$, più $m_{h}-1$ funzioni dei $\rho_{h_{i}}\left(i=2,3, \ldots m_{h}\right)$, che si possono quindi sostituire a questi ultimi. Successivamente si possono in egual modo scegliere gli integrali $\rho_{h_{t}}\left(i=3,4, \ldots m_{h_{i}}\right)$ in modo che siano ortogonali a $\rho_{h_{1}}$ e $\rho_{h_{2}}$ e proseguendo collo stesso metodo scegliere gli integrali del gruppo $g_{h}$ e così quelli di qualunque altro gruppo in modo che siano ortogonali fra di loro due a due. Dopo aver fatto ciò avremo costruito un sistema di $n-1$ integrali della (I) ortogonali fra di loro due a due nella varietà di elemento lineare $\varphi$. Possiamo dunque concludere che:

Le condizioni necessarie e sufficienti perchè la equazione (I) nella varietò di elemento lineare $\varphi$ ammetla un sistema di $n-1$ integrali ortogonali fra di loro due a due consistono in ciò che per ogni valore $\omega_{\text {¿della equazione }}$ algebrica caratteristica multipla del grado $m_{h}$ determinati $m_{h}$ sistemi indipendenti di soluzioni $\Xi_{n_{i}}\left(i=1,2, \ldots m_{i}\right)$ delle equazioni $(2)$ del $\$ 2$ e posto $\mathrm{H}_{r h_{i}}=\underset{10^{\circ} c_{r s} \Xi_{s h_{i}} \text { Siano }}{\mathbf{S}}$

uno qualunque dei sistemi:

$$
\sum_{r} \mathrm{H}_{r h_{i}} \psi^{(\boldsymbol{r})}=0 \quad\left(i=1,2, \ldots m_{h}\right)
$$

2. ${ }^{\circ}$ Siano completi $i$ sistemi di equazioni, che risultano della (I) e di due qualunque di questi ultimi sistemi.

Analiticamente queste condizioni si esprimono, come si dimostra con metodo analogo a quello tenuto nel $\S 3$, mediante $\mathrm{i}$ due sistemi di equazioni:

$$
\begin{aligned}
& \mathbf{\Sigma}_{r s}\left(Y_{s} \mathrm{H}_{, h_{i}}^{(s)}-\mathrm{H}_{s h_{i}} Y_{r}^{(s)}\right) \Xi_{r k_{i}}=0 \\
& \mathbf{\Sigma}_{r s}\left(\mathrm{H}_{s h_{i}} \mathrm{H}_{i k_{j}}^{(s)}-\mathrm{H}_{s k_{j}} \mathrm{H}_{r h_{i}}^{(s)}\right) \Xi_{r l_{g}}=0,
\end{aligned}
$$

che si possono anche trasformare nei:

$$
\begin{aligned}
& \mathbf{\Sigma}_{r s} Y_{r} \mathrm{H}_{s h_{s}}\left(\Xi_{r k_{j}}^{(s)}-\Xi_{s k_{i}}^{(r)}\right)=0 \\
& \boldsymbol{\Sigma}_{r s} \mathrm{H}_{r h_{i}} \mathrm{H}_{s k_{j}}\left(\Xi_{r l_{g}}^{(s)}-\Xi_{s l_{g}}^{(r)}\right)=0,
\end{aligned}
$$

e nei quali $h, k, l$ debbono essere differenti fra loro. Sempre analogamente a quanto si è fatto nel $\S 3$ si può vedere che il primo membro di una $\left(\Omega_{k k}\right)$ cambia soltanto di segno, scambiandovi $h_{i}$ con $k_{j}$. Le equazioni del sistema $\left(\Omega_{h k}\right)$ si dividono cosi in tanti gruppi quante sono le combinazioni due a due 
dei gruppi di radici eguali della equazione algebrica caratteristica, quello corrispondente alla combinazione dei due gruppi $G_{h}$ e $G_{k}$ contenendone $m_{h} m_{l}$. Quelle del sistema $\left(\Omega_{h k l}\right)$ si dividono invece in tanti gruppi quanto sono le combinazioni di quei gruppi tre a tre, quello corrispondente alla combinazione $G_{h} G_{k} G_{i}$ contenendone $3 m_{h} m_{k} m_{i}$.

Possiamo ancora aggiungere che:

Quando le condizioni sopra enunciate sono verificate gli integrali del sistema ortogonale non sono in generale completamente determinati. Essi si dividono in tanti gruppi $g_{h}$ corrispondenti ai gruppi di radici eguali della equazione algebrica caralteristica, il gruppo $g_{h}$ corrispondente al gruppo di tutle le radici eguali ad $\omega_{h}$ in numero di $m_{h}$ risultando di $m_{h}$ integrali indipendenti del sistema di equazioni, che risulta della proposta e di tutti $i$ gruppi della forma $\sum_{r} H_{2 k_{j}} \psi^{(r)}=0$, in cui sia $k$ differente da h. Entro a ciascun gruppo $g_{h}$, se $\grave{e} m_{h}>1$, la scelta degli integrali è possibile in infiniti modi.

Così il sistema di integrali ortogonali non è unico e determinato se non nol caso, in cui la equazione algebrica caratteristica non ha radici multiple, nel qual caso per la sua determinazione si applica il teorema del $\S 3$.

\section{$\S 5$.}

Sul problema dei sistemi ortogonali nelle varietà ad $n$ dimensioni.

Se si suppone che le quantità $X_{r}$ siano proporzionali alle derivate $p^{(r)}$ di una funzione $\rho$, il problema, che trattiamo, si riduce a quello di determinare le condizioni necessarie e sufficienti perchè nella varietà qualunque di elemento lineare $\varphi$ il sistema di luoghi di equazioni $\rho=$ costante appartenga ad un sistema $n^{u p l o}$ ortogonale e, quando queste condizioni siano verificate, di trovare i parametri degli $n-1$ sistemi di luoghi, che col proposto costituiscono il detto sistema; problema già risolulo dal Darboux per $n$ qualunque, ma limitatamente al caso in cui la espressione di $\varphi^{2}$ sia $\sum_{i}^{n} d x_{i}^{2}$.

Se per le quantità $X_{r}$. si prendono precisamente lo $p^{(r)}$, le $Y_{r}$ ed $X_{r s}$, coincidono colle espressioni, per cui nella mia Memoria: Sui parametri e gli invarianti delle forme differenziali quadratiche adottai i simboli pr e prs. Conserverò qui le stesse notazioni così che avremo:

$$
\begin{aligned}
& \rho_{r}=\mathbf{\Sigma}_{s} c_{r s} \rho^{(s)} \\
& \rho_{r s}=p^{(r s)}-\mathbf{\Sigma}_{i} a_{r s, i} \rho_{i},
\end{aligned}
$$


e la equazione algebrica caratteristica presa sotto la forma $\left(e_{1}\right)$ sarà:

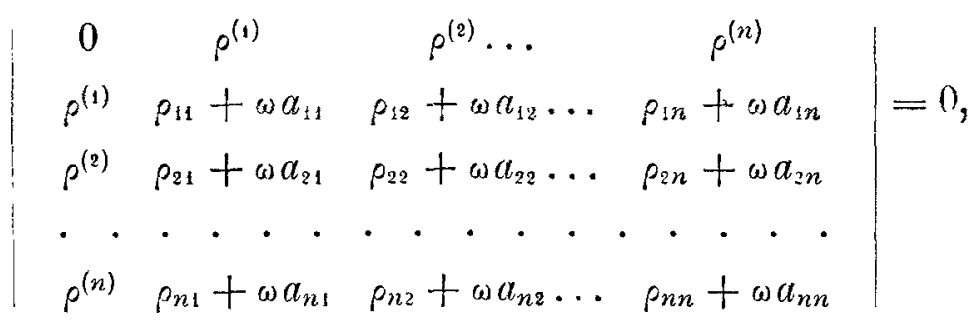

mentre le equazioni (2) del $\S 2$ diventeranno:

posto:

$$
\left.\begin{array}{c}
\boldsymbol{\Sigma}_{r} \rho_{r} \Xi_{r h}=0 \\
\rho_{s} \Xi_{o h}+\boldsymbol{\Sigma}_{r}\left(\rho_{r s}^{\prime}+\omega_{h} c_{r s}\right) \Xi_{, h}=0,
\end{array}\right\}
$$

$$
\rho_{r s}^{\prime}=\Sigma_{h k} \rho_{i k} c_{l r} c_{k s} \text {. }
$$

Notiamo ancora che sempre nel caso, che consideriamo, le equazioni di due gruppi:

$$
\mathbf{\Sigma}_{r} \mathrm{H}_{r h_{i}} \psi^{(r)}=0 \quad \mathbf{\Sigma}_{r} \mathrm{H}_{r k_{j}} \psi^{(r)}=0
$$

corrispondenti secondo il $\S 4$ a due gruppi $G_{h}$ e $G_{k}$ di radici della equazione $(P)$, che comprendono tutte quelle eguali rispettivamente ad $\omega_{h}$ ed $\omega_{k}$, hanno comuni non soltanto gli integrali della:

$$
\Sigma_{r} \operatorname{pr}_{r} \psi^{(r)}=0
$$

appartenenti ai gruppi, che corrispondono agli altri gruppi di radici di $(P)$, ma anche $\rho$, come si desume dalla prima delle (3), a cui si può dare la forma $\Sigma_{r} \mathrm{H}_{r h} \rho^{(s)}=0$, posto al solito:

$$
H_{, h}=\Sigma_{s} c_{r s} \Xi_{s h}
$$

Esse costituiscono quindi di per sè, cioè senza bisogno di associarsi la (I), un sistema completo e l'enunciato del teorema del $\S 4$ si può in questo caso modificare come segue:

Le condizioni necessarie e sufficienti perchè il sistema di luoghi di equazioni $\rho\left(x_{1} x_{2} \ldots x_{n}\right)=\rho$ appartenga ad un sistema $n^{\text {uplo }}$ ortogonale nella varietà, per cui il quadrato dell'elemento lineare è $\varphi^{2}=\sum_{1}^{n} a_{r s} d x_{r} d x_{s}$, consistono in ciò che per ogni radice $\omega_{h}$ della equazione $(P)$ multipla del grado $m_{h}$ determinati $m_{h}$ sistemi indipendenti $\Xi_{r h_{1}}\left(i==1,2, \ldots m_{h}\right)$ di soluzioni delle 
equazioni (3) e costruiti $i$ sistemi di equazioni:

$$
\Sigma_{r} \mathrm{H}_{2 \cdot h_{1}} \psi^{(r)}=0 \quad\left(i=1,2, \ldots m_{k}\right)
$$

le $\mathrm{I}_{3} h_{2}$ essendo date dalle (5), siano completi: $10^{\circ} i$ sistemi di equazioni che risultano della $\boldsymbol{\Sigma}_{r} \rho_{r} \psi^{(r)}=0$ e di uno qualunque dei sistemi $\left(\mathrm{I}_{h}\right) ; 2 .{ }^{\circ} i$ sistemi di equazioni, che risultano di due sistemi $\left(\mathrm{I}_{h}\right)$ qualunque.

Verificate queste condizioni $i$ parametri dei sistemi di luoghi, che assieme al sistema dato $(\rho)$ costituiscono il sistema $n^{\text {uplo }}$ ortogonale nella varietà di elemento lineare $\varphi$, non sono in generale completamente determinati. Essi si dividono in tanti gruppi $\left(g_{h}\right)$ corrispondenti ai gruppi di radici della equazione $(P)$ formati con tutte quelle, che hanno uno stesso valore $\omega_{h}$, ogni gruppo $\left(g_{h}\right)$ contenendone $m_{h}$, se questo $\grave{e} i l$ grado di multiplicitè di $\omega_{h}$, e precisamente $m_{h}$ integrali indipendenti del sistema di $n-m_{h}$ equazioni, che risulta della $\Sigma_{r} \rho_{r} \psi^{(r)}=0$ e dei sistemi $\left(\mathrm{I}_{k}\right)$ corrispondenti a radici della $(P)$ differenti $\overline{d a} \omega_{h}, i$ quali integrali per $m_{h}>1$ non sono quindi completamente determinati.

Non occorre riscrivere qui per questo caso speciale le equazioni trovate pel caso generale nel $\$ 4$ e che senza alcuna limitazione circa l'equazione $(P)$ esprimono analiticamente le condizioni necessarie e sufficienti sopra enunciate per la esistenza di un sistema $n^{\text {uplo }}$ ortogonale, di cui faccia parte $(\rho)$, nella varietà di elemento lineare $\varphi$. Lo faremo soltanto pel caso più comune, in cui la equazione $(P)$ non ha radici multiple e pel qualo valgono i risultati del $\S 3$. Dopo alcune riduzioni dalle (1) e (2) si ottengono le:

$$
\begin{aligned}
& Y_{p}^{(s)}=\rho_{p}^{(s)}=\mathbf{\Sigma}_{q} c_{p q} \rho_{q s}-\mathbf{\Sigma}_{h q} c_{p q} a_{h s q} \rho_{h} \\
& X_{i s}^{(p)}=\rho_{s}^{(p)}=p_{r s p}+\mathbf{\Sigma}_{h q} c_{h q}\left(a_{r p, q} \rho_{h s}+a_{s p, q} \rho_{h r}\right),
\end{aligned}
$$

posto ancora come nella citata Memoria:

$$
\begin{aligned}
& \left.\rho_{r s p}=p^{(r s p)}-\mathbf{\Sigma}_{h_{q}} c_{h_{q}}\left(a_{r p q} \rho_{h_{s}}+a_{s p q} \rho_{h r r}+a_{s r q} \rho_{h p}\right)\right) \\
& -\Sigma_{k}\left(a_{r y, k}^{(p)}-\mathbf{\Sigma}_{h_{q}} c_{h q} a_{k p h} a_{r, q, q}\right)_{\rho k} . \quad j
\end{aligned}
$$

Si ha per conseguenza dalle (17) del $\S 3$

$$
X^{\prime} \cdot s=-\frac{1}{2} \Sigma_{p} \rho_{r s p} \rho_{p}+\mathbf{\Sigma}_{p q} c_{p q} \rho_{p r} \rho_{q *} .
$$

Le (21) del $\S 3$ confrontate colle (6) di questo dànno:

$$
X_{r s, q}=\rho_{r q s}+\rho_{s q r}-\rho_{r s q},
$$


e queste alla loro volta:

$$
2 \rho_{s q r}=X_{r s, q}+X_{r q, s} .
$$

Siccome nel caso nostro le equazioni $\left(\Omega^{\prime}{ }_{h k i}\right)$ del $\S 3$, essendo $X_{q}^{(r)}=X_{r}^{(q)}$ si riducono alla forma:

$$
\mathbf{\Sigma}_{r s q} X_{r s, q} \mathrm{H}_{r l} \mathrm{H}_{s k} \mathrm{H}_{q i}=0
$$

così le (6), (7) ed (8) ci dicono che le equazioni $\left(\Omega_{h k}\right)$ ed $\left(\Omega_{h k i}\right)$ si possono in questo caso mettere sotto la forma:

$$
\begin{array}{cr}
\Sigma_{r s q} \rho_{r s q} p_{q} \mathrm{H}_{r h} \mathrm{H}_{s k}=2 \boldsymbol{\Sigma}_{p q r s} c_{p q} \rho_{p r} \rho_{q s} \mathrm{H}_{r h} \mathrm{H}_{s k} & \left(P_{h k}\right) \\
\sum_{r s q} \rho_{r s q} \mathrm{H}_{q i} \mathrm{H}_{r h} \mathrm{H}_{s k}=0, & \left(P_{h k i}\right)
\end{array}
$$

nelle quali $h, l i, i$ sono tre indici differenti scelti comunque tra $i$ numeri $1,2, \ldots n-1$.

E facile verificare che i sistemi di equazioni $\left(P_{l k}\right)$ e $\left(P_{h k i}\right)$ coincidono rispettivamente coi sistemi (142) e (145) della Seconda Parte della Memoria del sig. Darboux: Sur la Thíorie des coordonnées curvilignes et des systhèmes orthogonaux nel caso che $q^{2}$ abbia la forma $\sum_{1}^{n} d x_{1}^{2}$.

Notiamo che le espressioni $\rho_{r s}$, $\rho_{r s q}$ sono quelle, che a tenore della mia Memoria più volte citata giova considerare rispettivamente invece delle derivate seconde e terze di $p$.

Se si pone:

$$
\begin{aligned}
& 3_{a_{r s q}}=\rho_{r s q}+\rho_{r q s}+\rho_{s q r} \\
& \beta_{r s, q}=\rho_{r s q}-\rho_{r q s},
\end{aligned}
$$

si hanno anche 10:

$$
\begin{aligned}
& \beta_{s r . q}=\rho_{r s q}-\rho_{s q r} . \\
& 3 \rho_{r q q}=3 a_{r s q}+\beta_{r s, q}+\beta_{s r, q},
\end{aligned}
$$

le quali ci dicono che al sistema $\left(P_{h k i}\right)$ si possono sostituire i due:

posto:

$$
\begin{aligned}
& \sum_{r s q} \alpha_{r s q} \mathrm{H}_{r h} \mathrm{H}_{s h} \mathrm{H}_{q i}=0 \\
& \sum_{s r p q} a_{p r, q s} \rho_{p} \mathrm{H}_{q i} \mathrm{H}_{r h} \mathrm{H}_{s h}=0,
\end{aligned}
$$

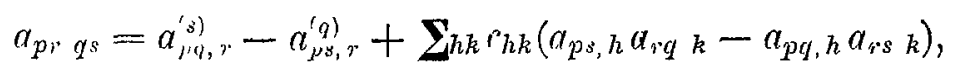

poichè i:

$$
\beta_{s r, q}=\boldsymbol{\Sigma}_{p} u_{r p q s} \rho_{p}
$$


Poichè il numero delle equazioni $\left(P_{h k_{i}}\right)$ indipendenti fra loro è

$$
\frac{(n-1)(n-2)(n-3)}{2}
$$

c quello delle $\left(P^{\prime}{ }_{h k i}\right)$, come si vede subito, è $\frac{(n-1)(n-2)(n-3)}{2 \cdot 3}$ quello delle $\left(P^{\prime \prime}{ }_{h k i}\right)$ sarà

$$
\frac{(n-1)(n-2)(n-3)}{2}-\frac{(n-1)(n-2)(n-3)}{2 \cdot 3}=\frac{(n-1)(n-2)(n-3)}{3} .
$$

Cio si deduce anche direttamente dall'osservare che esistono le identità:

$$
a_{p r, q s}+a_{p q, s r}+a_{p s, q r}=0 \text {. }
$$

Osservando ancora che il numero delle equazioni $\left(P_{h k}\right)$ è $\frac{(n-1)(n-2)}{2}$ e ricordando le cose dimostrate nel $\S 3$ noi possiamo concludere:

Data una funzione $p$ delle coordinate $x_{1}, x_{2}, \ldots x_{n}$ di una varietà ad $n$ dimensioni, per cui il quadrato dell'elemento lineare è $\varphi^{2}=\mathbf{\Sigma}_{r s} a_{r s} d x_{r} d x_{s}$, indicando con a il discriminante $d i \varphi^{2}$, le quantità $\rho_{q}, \rho_{r s}, a_{r s q}, a_{p r, q s}$ essendo quelle definite dalle equazioni (1) (2) (6) (7) ed (11), posto:

$$
\begin{gathered}
X(\omega)==\mathbf{\Sigma} \pm\left(\rho_{11}+\omega a_{11}\right)\left(\rho_{22}+\omega a_{22}\right) \cdots\left(\rho_{n n}+\omega a_{n n}\right) \\
H_{r h}=\frac{1}{a} \boldsymbol{\Sigma}_{i} \rho^{(i)} \frac{d X(\omega h)}{d\left(\rho_{i r}+\omega h a_{i r}\right)},
\end{gathered}
$$

dove wh è una radice della equazione:

$$
\sum r s \frac{d X(\omega)}{d\left(\rho r s+\omega a_{r s}\right)} \rho^{(r)} \rho^{(s)}=\hat{v},
$$

qualora questa non abbia radici multiple, le condizioni necessarie e sufficienti perchè il sistema di luoghi di parametro $\rho$ appartenga ad un sistema $n^{u p l o}$ ortogonale nella varietà di elemento lineare $\varphi$ sono che $\rho$ soddisfi: $10^{\circ}$ ad un sistema di $\frac{n(n-1)(n-2)}{2 \cdot 3}$ equazioni a derivate parziali di $3 .^{\circ}$ ordine, il quale risulta dalle $\left(P_{h k}\right)$ e dalle $\left(P_{h k i}^{\prime}\right) ; 2 .^{\circ}$ ad un sistema di $\frac{(n-1)(n-2)(n-3)}{3}$ equazioni a derivate parziali di $2 .^{\circ}$ ordine, che sono le $\left(P_{h k i}^{\prime \prime}\right)$.

Verificate queste condizioni le $n-1$ equazioni a differenziali totali:

$$
\Sigma_{r} \Xi_{r h} d x_{r}=0 \quad(h=1,2, \ldots n-1)
$$




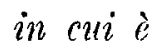

$$
\Xi_{r h}=\Sigma_{s} c_{r s} \mathrm{H}_{s h}
$$

sono integrabili ciascuna mediante una sola primitiva $p_{h}\left(x_{1}, x_{2}, \ldots x_{n}\right)=p_{h} e$ $\rho_{1}, \rho_{2}, \ldots \rho_{n-1}$ sono $i$ parametri dei sistemi di luoghi, che con quello di parametro $p$ costituiscono un sistema $n^{u p l a}$ ortogonale nella varietì di elemento lineare $\varphi$.

Le equazioni di $2 .^{\circ}$ ordine del sistema $\left(P_{h k i}^{\prime \prime}\right)$ possono per la natura speciale della varietà ad $n$ dimensioni ridursi ad un numero minore di quello sovra indicato, il che fa vedere come il grado di difficoltà per la esistenza dei sistemi ortogonali in una varietà data non dipenda soltanto dal numero delle dimensioni, ma anche dalla natura di questa. Fermo il numero $n$, questa difficoltà è la minore possibile se $\varphi^{2}$ può ridursi alla forma $\sum_{i}^{n} d x_{r}^{2}$, cioè se la varietà è piana od euclidea, poichè allora, come si sa, i coefficienti $a_{r p, q s}$ sono identicamente nulli e il sistema di equazioni di $2 .^{\circ}$ ordine $\left(P^{\prime \prime}{ }_{k k i}\right)$ viene meno del tutto.

Se invece la espressione $\varphi^{2}$ del quadrato dell'elemento lineare della data varietà può dedursi dalla $\sum_{1}^{n+1} d y_{i}^{2}$ ponendo per $y_{1}, y_{2}, \ldots y_{n+1}$ delle convenienti funzioni di $x_{1}, x_{2}, \ldots x_{n}$ ed anche in un caso più generale $\left({ }^{*}\right)$ i coefficienti $a_{r p, s q}$ prendono la forma $(p q)(r s)-(p s)(r q),(r s)$ essendo gli elementi di un determinante simmetrico di grado $n$. Le equazioni $\left(P_{k k i}^{\prime \prime}\right)$ prendono dunque in questo caso la forma:

$$
\mathbf{\Sigma}_{p q}(p q) \rho_{p} \mathrm{H}_{q i} \mathbf{\Sigma}_{r s}(r s) \mathrm{H}_{r h} \mathrm{H}_{s k}=\Sigma_{p q}(p q) \rho_{p} \mathrm{H}_{q k} \mathbf{\Sigma}_{r s}(r s) \mathrm{H}_{r h} \mathrm{H}_{s i}
$$

ovvero l'altra:

$$
\Sigma_{r s}(r s) \mathrm{H}_{r h} \mathrm{H}_{s k}=\alpha_{h} \alpha_{k} \quad(h \lesseqgtr k, h, k=1,2, \ldots n),
$$

posto $p_{r}=\mathrm{H}_{r n}$, ed indicando con $\alpha_{1}, \alpha_{2}, \ldots \alpha_{n}$ delle quantità arbitrarie. In questo caso adunque le equazioni $\left(P_{h k i}^{\prime}\right)$ si riducono ad $\frac{n(n-1)}{2}-n=\frac{n(n-3)}{2}$ distinte.

(*) Veggasi a questo proposito il $\$ 3$ della mia Memoria dal titolo: Principii di una teoria delle forme differenziali quadratiche nel Tomo XII della Serie 2.2 di questi Annali. 
$\S 6$.

\section{Applicazione dei risultati generali al caso di $n=3$.}

Proponiamoci sulla scorta dei risultati ottenuti nei precedenti paragrafi di riconoscere se una equazione:

$$
\sum_{1}^{3} Y_{r} \psi(r)=0
$$

ammette due integrali ortogonali fra di loro nella varietà, per cui il quadrato dell' elemento lineare è:

$$
\varphi^{2}=\sum_{1}^{3} r s a_{r s} d x_{r} d x_{s}
$$

In questo caso se si conviene di considerare come eguali le quantità, i cui indici sono congrui fra loro rispetto al modulo 3 e si pone:

$$
\mathrm{H}_{r}(\omega)=\frac{1}{a}\left|\begin{array}{lll}
X_{r} & X_{r, r+1}+\omega a_{r, r+1} & X_{r, r+2}+\omega a_{r, r+2} \\
X_{r+1} & X_{r+1, r+1}+\omega a_{r+1, r+1} & X_{r+1}+2+\omega a_{r+1, r+2} \\
X_{r+2} & X_{r+2, r+1}+\omega a_{r+2} r+1 & X_{r+2, r+2}+\omega a_{r+2, r+2}
\end{array}\right|,
$$

sostituendo per le $X_{r}$ le loro espressioni in funzione delle $Y_{r}$ si trova:

$$
\mathrm{H}_{r}(\omega)=\omega^{2} Y_{r}+\omega Y_{r} \sum_{s t} c_{s t} X_{s t}-\omega \Sigma_{t} Y_{t} \Sigma_{s} c_{r s} X_{s t}-\frac{1}{a} \frac{d M}{d X_{r}},
$$

posto:

$$
M=\left|\begin{array}{cccc}
0 & X_{1} & X_{2} & X_{3} \\
X_{1} & X_{11} & X_{12} & X_{13} \\
X_{2} & X_{21} & X_{22} & X_{23} \\
X_{3} & X_{31} & X_{32} & X_{33}
\end{array}\right|
$$

Poichè la equazione algebrica caratteristica sotto la forma $\left(e_{i}\right)$ equivale alla:

posto:

$$
\sum_{r} H_{r}(\omega) X_{r}=0 \text {, }
$$

$$
\Lambda=\frac{\sum_{s t} X_{s t} Y_{s} Y_{t}}{\sum_{r} X_{r} Y_{r}}, \quad N=\sum_{s t} c_{s t} X_{s t}
$$

essa si può mettere sotto la forma:

$$
\omega^{2}+(N-\Lambda) \omega-\frac{M}{a}=0 \text {. }
$$


Se le radici $\omega_{1}$ ed $\omega_{2}$ di questa non sono eguali fra di loro, si ha seguendo la notazione adottata nel caso generale:

e dalle (1):

$$
\mathrm{I}_{1} h=\mathrm{H}_{r}\left(\omega_{h}\right) \quad(h=1,2, r=1,2,3),
$$

$$
\mathrm{H}_{r h}=\omega_{h}^{2} Y_{\cdot}+\omega_{h} N Y_{\mathbf{r}}-\omega_{h} \mathbf{\Sigma}_{t} Y_{t} \mathbf{\Sigma}_{s} c_{r s} X_{s t}-\frac{1}{a} \frac{d M}{d \mathbf{X}_{r}}
$$

e per $\omega_{n}^{2}$ sostituendo il valore dato dalla $(E)$ :

$$
\mathrm{H}_{r h}=\omega_{h}\left(\Lambda Y_{r}-\mathbf{\Sigma}_{t} Y_{t} \mathbf{\Sigma}_{s} c_{s r} X_{s t}\right)+\frac{1}{a}\left(M Y_{r}-\frac{d M}{d X_{r}}\right)
$$

Da questa poi si traggono le:

$$
\Xi_{, h}=\Sigma_{s} a_{r s} \amalg_{s h}=\omega_{h}\left(\Lambda X_{r}-\Sigma_{t} X_{r t} Y_{t}\right)+\frac{1}{a}\left(M X_{r}-\Sigma_{s} a_{r s} \frac{d M}{d X_{s}}\right)
$$

Per $n=3$ le equazioni di condizione per la esistenza di due integrali della (I) ortogonali nella varietà di elemento lineare $\varphi$ già trovate pel caso generale nel $\S 3$ si riducono ad una sola, la quale costituisce il sistema $\left(\Omega_{h k}\right)$ e i risultati di quel paragrafo, riferendosi alle notazioni già stabilite prendono la forma seguente:

La condizione necessaria e sufficiente perchè la equazione (I) ammetta due integrali ortogonali nella varietà di elemento lineare $\varphi$, qualora la equazione algebrica $(E)$ non abbia le radici eguali, è espressa da una equazione a derivate parziali di $2 .^{\circ}$ ordine rispetto ai coefficienti dell'equazione e del quadrato dell'elemento lineare. Quando questa sia soddisfatta le due equazioni a differenziali totali:

$$
\sum_{1}^{3} \Xi_{r} h d x_{r}=0 \quad(h=1,2),
$$

sono integrabili amendue con una sola primitiva $\rho_{h}\left(x_{1} x_{2} x_{3}\right)=\rho_{h} e_{0} \rho_{1}, \rho_{2}$ sono gli integrali ortogonali cercati.

La equazione a derivate parziali sopra accennata ̀̀ suscettibile di diverse forme, che si hanno dalle $\left(\Omega_{h k}\right),\left(\Omega_{h k}^{\prime}\right)$ ed $\left(\Omega^{\prime \prime}{ }_{h k}\right)$ del $\$ 3$ ponendovi per le $\Xi_{r h}$ od $\mathrm{H}_{r h}$ le espressioni (3') o (3) sopra determinate. Essa è suscettibile anche delle due forme

$$
\mathbf{\Sigma}_{r} \Xi_{i \cdot h}\left(\Xi_{i+1, h}^{\left(r+y^{\prime}\right)}-\Xi_{r+2, h}^{(\prime+1\}}\right)=0, \quad(h=1,2),
$$

che vengono dalla applicazione di un noto teorema del Calcolo differenziale.

Altre forme della stessa equazione si ottengono mediante altre espressioni delle $\Xi_{r h}$ ed $U_{r h}$, che si trovano nel modo seguente. Tra le equazioni (11') 
del $\$ 3$ prendiamo le:

$$
\begin{aligned}
& \sum_{r}^{+2} \mathrm{X}_{t} \mathrm{H}_{t h}=0 \\
& \sum_{r}^{r+2}\left(X_{t, r+1}+\omega_{h} a_{t r+1}\right) \mathrm{H}_{t h}=-X_{r+1} \mathrm{H}_{0 h} \\
& \sum_{r}^{r+2}\left(X_{t, r+2}+\omega_{h} a_{t, r+2}\right) \mathrm{H}_{t h}=-X_{r+2} \mathrm{H}_{0 h} .
\end{aligned}
$$

Queste risolute rispetto alle $\mathrm{II}_{l h}$ dànno:

$$
\frac{1}{\mathbf{H}_{0 / h}} \mathrm{H}_{r l l} \mathrm{H}_{t h}=\omega_{h}\left(c_{r t} \mathbf{\Sigma}_{u} X_{u} Y_{u}-Y_{r} Y_{t}\right)-\frac{1}{a} \cdot \frac{d M}{d X_{r t}}
$$

dalle quali moltiplicate per $a_{s t}$, sommando poi rispetto a $t$ si traggono le:

$$
\frac{1}{\mathrm{I}_{0 h}} \mathbf{H}_{r l} \Xi_{s h}=\omega_{h}\left(\varepsilon \mathbf{\Sigma}_{u} X_{u t} Y_{u b}-Y_{r} X_{s}\right)-\frac{1}{a} \mathbf{\Sigma}_{t} a_{s t} \frac{d M}{d X_{r t}},
$$

in cui è $\varepsilon=1$ per $s \equiv r(\bmod .3)$ ed $\varepsilon=0$ in ogni altro caso. Notando ancora che ̀̀:

$$
\Sigma_{t} a_{s t} \frac{d M}{d X_{r t}}=\Sigma_{t} Y_{t}\left|\begin{array}{ccccc}
0 & 0 & X_{r+1} & X_{r+2} \\
a_{r t} & a_{r s} & X_{r, r+1} & X_{r, r+2} \\
a_{r+1, t} & a_{r+1, s} & X_{r+1, r+1} & X_{r+1, r+2} \\
a_{r+2, t} & a_{r+2, s} & X_{r+2, r+1} & X_{r+2, r+2}
\end{array}\right|,
$$

che nella sommatoria del secondo membro basta fare $t=s+1$ e $t=s+2$, mediante alcune riduzioni, indicando con $\omega_{k}$ la radice della $(E)$ differente da $\omega_{h}$, si trova:

$$
\begin{gathered}
\frac{1}{\mathrm{~B}_{0 h}} \mathrm{I}_{r \cdot h} \Xi_{s h}=-\varepsilon \omega_{k} \mathbf{\Sigma}_{t} X_{t} Y_{t}-\left(\Lambda-\omega_{k}\right) Y_{r} X_{s}-\mathbf{\Sigma}_{t} X_{t} Y_{t} \mathbf{\Sigma}_{u} c_{r u} X_{u s} \\
+X_{s} \sum_{t} c_{r t} P_{t}+Y_{r} P_{s}
\end{gathered}
$$

posto:

$$
P_{s}=\Sigma_{t} X_{s t} Y_{t}
$$

Se la equazione $(E)$ ha le radici eguali, il loro valore comune annulla, come è stato detto, i minori di $1 .^{\circ}$ ordine dei determinanti, che costituiscono il primo membro della equazione stessa presa sotto la forma $(e)$ od $\left(e_{1}\right)$. Prendendola sotto la forma $\left(e_{1}\right)$ uno stesso valore di $\omega$ deve adunque soddisfare alle 
tre equazioni, che si hanno dalla:

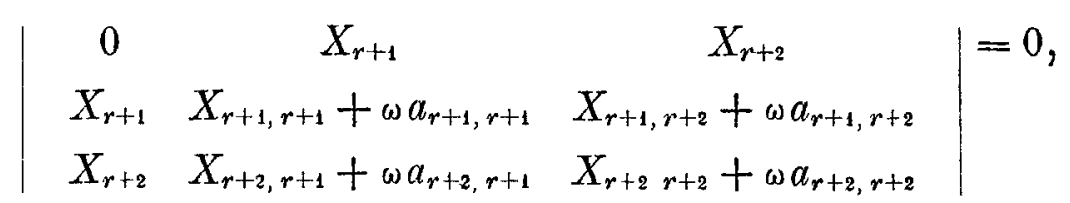

facendo $r=1,2,3$, e che si possono anche mettere sotto la forma:

$$
a \omega=\frac{\frac{d M}{d X_{r r}}}{c_{r r} \sum_{t} X_{t} Y_{t}-Y_{r}^{2}} \quad(r=1,2,3) .
$$

Possiamo dunque asserire che:

Le condizioni necessarie e sufficienti perchè la equazione $(E)$ abbia le radici eguali sono date dalle equazioni (c), in cui w rappresenta una indeterminata. Quando le due equazioni, che risultano dalla eliminazione di w tra le (c) sono identicamente soddisfatte, ad ogni integrale della equasione (I) ne corrisponde un altro, che gli ̀̀ ortogonale nella varietà di elemento lineare $\varphi$.

Di questo teorema sarebbe facile dare una dimostrazione speciale diretta.

Se $\varphi$ è l'elemento lineare dello spazio euclideo in coordinate cartesiane ortogonali, si ha:

$$
\begin{gathered}
a=1, \quad Y_{r}=X_{r}, \quad X_{r s}=\frac{X_{r}^{(s)}+X_{s}^{(r)}}{2}, \quad \Lambda=\frac{\sum_{r s} X_{r s} X_{r} X_{s}}{\sum_{r} X_{r}^{2}} \\
N=\sum_{r} X_{r r} \\
X_{r s}^{\prime}=Y_{r s}^{\prime}=\Sigma_{p} X_{p r} X_{p s}+\frac{1}{2} \sum_{p}\left(X_{p r} X_{s}^{(p)}+X_{p s} X_{r}^{(p)}-X_{p} X_{r s}^{(p)}\right)_{2}
\end{gathered}
$$

e le espressioni sopra trovate per le $\mathrm{H}_{r h}$ e $\Xi_{r h}$ dànno:

$$
\begin{gathered}
\mathrm{H}_{r h}=\Xi_{r h}=\omega_{h}\left(P_{r}-\Lambda X_{r}\right)+\frac{d M}{d X_{r}}-M X_{r} \\
\frac{1}{\Xi_{0 h}} \Xi_{r h} \Xi_{s h}=-\varepsilon \omega_{k} \mathbf{\Sigma}_{t} X_{t}^{2}-\left(\Lambda-\omega_{k}\right) X_{r} X_{s}-X_{r s} \sum_{t} X_{t}^{2}+X_{s} P_{r}+X_{r} P_{s} .
\end{gathered}
$$

Se, come può sempre farsi, si suppone che sia:

$$
\Sigma_{t} X_{t}^{2}=1
$$

si ha:

$$
\Sigma_{t} X_{t} X_{i}^{(v)}=0, \quad \Lambda=0, \quad P_{r}=\frac{1}{2} \Sigma_{t} X_{t} X_{r}^{(t)}
$$


e alle precedenti può darsi facilmente la forma:

$$
\begin{gathered}
\frac{1}{\Xi_{0 / h}} \Xi_{r h} \Xi_{s h}=-\varepsilon_{\omega_{k}}-X_{r}^{(s)}+L_{r k} X_{s}+\frac{X_{r}^{(s)}-X_{s}^{(r)}}{2}+ \\
+\boldsymbol{\Sigma}_{t} X_{t}\left(X_{r} \frac{X_{s}^{(t)}-X_{t}^{(s)}}{2}-X_{s} \frac{X_{r}^{(t)}-X_{t}^{(r)}}{2}\right)
\end{gathered}
$$

essendo:

$$
L_{r k}=\Sigma_{t} X_{t} X_{r}^{(t)}+\omega_{k} X_{r},
$$

od anche sviluppando per $s=r, r+1, r+2$ :

$$
\begin{aligned}
& \frac{1}{\Xi_{0, h}} \Xi_{r h} \Xi_{r h}=-X_{r}^{(r)}+L_{r k} X_{r}-\omega_{h} \\
& \frac{1}{\Xi_{0 h}} \Xi_{r h} \Xi_{r+1, h}=-X_{r}^{(r+4)}+L_{r k} X_{r+1}-Q X_{r+2} \\
& \frac{1}{\Xi_{0 h}} \Xi_{r h} \Xi_{r+2, h}=-X_{r}^{(r+2)}+L_{r h} X_{r+2}+Q X_{r+1}
\end{aligned}
$$

posto:

$$
Q=\frac{1}{2} \mathbf{\Sigma}_{s} X_{s}\left(X_{s+2}^{(s+1)}-X_{s+1}^{(s+2)}\right)
$$

In queste $\omega_{h}$ ed $\omega_{k}$ sono le radici, che supponiamo disuguali, della equazione:

$$
\omega^{2}+N \omega-M=0 \text {. }
$$

Le equazioni $(c)$ prendono nel caso, che consideriamo, la forma:

$$
\frac{d M}{d \bar{X}_{r r}}=\omega\left(X_{r+1}^{2}+X_{r+9}^{2}\right)
$$

ovvero, sostituendo ai primi membri i loro sviluppi:

$$
\left(X_{r+1}^{(r+2)}+X_{r+2}^{(r+1)}\right) X_{r+1} X_{r+2}=X_{r+2}^{(r+2)} X_{r+1}^{2}+X_{r+1}^{(r+1)} X_{r+2}^{2}+\omega\left(X_{r+1}^{2}+X_{r+2}^{2}\right) \text {. }
$$

Supponiamo, come pud sempre farsi, che sia $X_{3}=1$. Queste equazioni prendono allora la forma:

$$
\begin{aligned}
& X_{1} X_{2}\left(X_{1}^{(2)}+X_{2}^{(1)}\right)=X_{2}^{2} X_{1}^{(1)}+X_{1}^{2} X_{2}^{(2)}+\omega\left(X_{1}^{2}+X_{2}^{2}\right) \\
& X_{2} X_{2}^{(3)}=X_{2}^{(2)}+\omega\left(1+X_{2}^{2}\right) \\
& X_{1} X_{1}^{(3)}=X_{1}^{(3)}+\omega\left(1+X_{1}^{2}\right) .
\end{aligned}
$$

Servendosi delle ultime due per calcolare i valori di $X_{1}^{13)}$ ed $X_{2}^{13,}$ e della prima 
per calcolare quello di $\omega$ si trova:

$$
\begin{gathered}
\left.\left(X_{1}^{2}+X_{2}^{2}\right)\left(X_{1} X_{2}^{(3)}-X_{2} X_{1}^{(3)}+X_{1}^{2)}-X_{2}^{(1)}\right)=2 X_{1} X_{2}\left(X_{2}^{(2)}-X_{41}^{(1)}\right)+\right\} \\
+2\left(X_{1}^{2} X_{1}^{(2)}-X_{2}^{2} X_{2}^{(1)}\right) .
\end{gathered}
$$

Poichè la condizione necessaria e sufficiente perchè $X_{1}, X_{2}, 1$ siano proporzionali alle derivate di una stessa funzione rispetto ad $x_{1}, x_{2}, x_{3}$ è data dall' annullarsi del primo membro di questa equazione e in tal caso, come è noto, $\mathrm{i}$ coefficienti della (I) sono proporzionali alle derivate del parametro di un sistema di sfere concentriche, possiamo dalla equazione (7) concludere che, anche limitandoci a considerare la varietà euclidea a tre dimensioni, esistono, oltre alla ricordata, altre equazioni a derivate parziali di $10^{\circ}$ ordine lineari ed omogenee tali, che ad ogni loro integrale ne corrisponde un altro, che gli è ortogonale.

Non mi tratterrò ad applicare le formule di questo paragrafo al caso, in cui $\varphi$ essendo l'elemento lineare di una varietà qualunque a tre dimensioni, $i$ coefficienti $X_{r}$ della equazione (I) sono proporzionali alle derivate di una stessa funzione rispetto alle $x_{r}$. Osserverò soltanto che le formole $\left(6^{\prime}\right)$, da cui scompaiono in questo caso i termini moltiplicati per $Q$, sono quelle che conducono alla equazione, a cui soddisfa il parametro di una famiglia di superficie appartenente ad un sistema triplo ortogonale nello spazio euclideo ed in coordinate cartesiane ortogonali sotto la forma datalo da Weingarten nel vol. LXXXIII del Giornale di Borchardt.

Padova, giugrno 1886. 